\title{
Synthesis of Octabromoperylene Dianhydride and Diimides: Evidence of Halogen Bonding and Semiconducting Properties
}

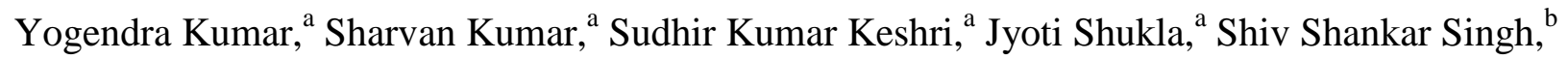 \\ Tejender S Thakur, ${ }^{\mathrm{b}}$ Mitchell Denti, ${ }^{\mathrm{c}}$ Antonio Facchetti ${ }^{\mathrm{c}, \mathrm{d}^{*}}$ and Pritam Mukhopadhyay ${ }^{\mathrm{a}^{*}}$ \\ ${ }^{a}$ Supramolecular and Material Chemistry Lab, School of Physical Sciences, Jawaharlal Nehru University, New Delhi- \\ 110067, India \\ ${ }^{b}$ Molecular and Structural Biology Divison, CSIR-CDRI, Lucknow-226031, India \\ ${ }^{c}$ Polyera Corporation, Skokie Illinois 60077, USA, ${ }^{d}$ Department of Chemistry, Northwestern University, Evanston, IL \\ 60208, USA
}

\section{Supporting Information Contents}

S. No. Topic

Page No.

1. Experimental section $\quad \mathrm{S} 2$

2. Synthesis and Characterization 4

3. Table S1: Comparison of the selected bond length, angle and torsion angle of 1 \& 2a S8

4. Table S2: Halogen Bonding interactions in 1 \& 2a 28

5. Table S3A-B: Optical and redox properties of the molecules S9

6. Table S4: Electron mobilities of the organic semiconductors $\quad$ S10

7. Figure S1: Cyclic voltammograms of 2a-e S11

8. Figure S2: Non-bonding interactions and self-assembly characteristics of $1 \quad S 12$

9. Figure S3-S4: Non-bonding interactions and self-assembly characteristics of 2a $\quad$ S12

10. Figure S5: Electronic density contours of the HOMO and LUMO of 2a S13

11. Figure S6: EPR spectra of the radical anion 2a-- S13

12. Figure S7: UV-Vis-NIR absorption characteristics of 2a-- S14

13. Figure S8: Thin film characteristics of the organic semiconductors S14

14. Figure S9: Device architecture and I-V plots of the organic semiconductors S15

15. Figure S10: FTIR spectra of 2a-e $\quad$ S15

16. Figure S11-S20: MALDI-TOF, HR-ESI-MS spectra S16

17. Figure S21-S38: ${ }^{1} \mathrm{H},{ }^{13} \mathrm{C}$ and COSY NMR spectra $\quad$ S20

$\begin{array}{llr}\text { 18. } & \text { References } & \text { S29 }\end{array}$ 


\section{Experimental Section}

General: Chemicals were sourced either from Sigma-Aldrich, Spectrochem India, Loba-chemie, or Thomas-Baker-India and were used as received. Thin layer chromatography (TLC) was carried out on aluminum plates coated with silica gel mixed with fluorescent indicator and was sourced from Merck, Germany. NMR $\left({ }^{1} \mathrm{H},{ }^{13} \mathrm{C}\right)$ spectra were recorded on a Bruker $500 \mathrm{MHz}$ spectrometer in $\mathrm{CDCl}_{3}$ or DMSO or THF with TMS as a standard. Spin multiplicities are reported as a singlet (s), doublet (d), and triplet (t) with coupling constants $(J$ ) given in Hz, or multiplet (m). MALDI-TOF mass spectral data were obtained using a Bruker made Auto flex TOF/TOF instrument with laser repetition rate of 50 psec. $\alpha$-Cyano-4hydroxycinnamic acid and 1,8,9-Anthracenetriol were used as the matrix for MALDI-TOF mass spectrometry. HR-ESI-MS spectral data were obtained using a waters make ESI-MS model Synapt G2 high definition mass spectrometry.

UV-vis-NIR and IR Spectroscopy: UV-vis-NIR spectra were recorded on a JASCO V-670UV-vis-NIR Spectrophotometer. All the spectroscopic experiments were carried out in UV Grade $\mathrm{CH}_{2} \mathrm{Cl}_{2}$ or $\mathrm{MeCN}$ which was sourced from Spectrochem, India. Infrared spectra were recorded in $\mathrm{KBr}$ pellet using a Varian 3100 FT-IR instrument.

Cyclic and Differential Pulse Voltammetry (CV/DPV): CV and SWV were carried out using a computer controlled potentiostat (CHI 650C) and a standard three electrode arrangement that consisted of both platinum working and auxiliary electrodes and saturated calomel (SCE) as reference electrode. All electrochemical measurements were carried out in Ar-purged DCM with n-Bu $\mathrm{NPF}_{6}$ as the supporting electrolyte. CV studies of the molecules $\mathbf{1}$, 2a-e, 3 and $\mathbf{4}(0.5 \mathrm{mM})$ were performed in degassed DCM under Ar atmosphere and the scan rate for the measurements was typically 200-300 mV/s. DPV was carried out keeping peak amplitude $50 \mathrm{mV}$, peak width $0.01 \mathrm{sec}$, pulse period $0.05 \mathrm{sec}$ and increment $\mathrm{E}$ at $20 \mathrm{mV}$.

Theoretical calculations: The ground-state geometry optimization was carried out applying the density functional theory (DFT) with the Becke three-parameter ${ }^{1}$ hybrid exchange functional in concurrence with the Lee-Yang-Parr gradient-corrected correlation function (B3LYP functional) ${ }^{2}$ with the 6-311G(d,p) basis set as implemented in Gaussian 09W. ${ }^{3}$ All the geometries were optimized without any constrain. To reduce the calculation time axial group $\left(\mathbf{R}^{\prime}\right)$ of all molecules has been replaced by methyl group.

X-ray Crystallography: Crystals of 1 were grown in THF solution by slow evaporation method at $15^{\circ} \mathrm{C}$. The crystals were adequately stable under ambient conditions, losing the crystalline nature for long exposure to air. The reported data set was collected by mounting the crystal with paratone oil in a loop. Xray reflections were collected on Bruker D8 Quest diffractometer with CMOS detector using MoK $\alpha$ radiation, generated from the micro-focus sealed tube. Data collection was performed using $\varphi$ and $\omega$ - 
scans of $0.5^{\circ}$ steps at $100 \mathrm{~K}$. Cell determination, data collection and data reduction were performed with the help of Bruker APEX2 (version: 2.1-b24) software. The structure were solved by intrinsic phasing method (SHELXS-97) and refined by full-matrix least squares refinement method based on $\mathrm{F}^{2}$, using, SHELXL2014. A total of 17752 reflections were measured out of which 3174 were independent and 2245 were observed [I>2 $\sigma(\mathrm{I})]$ for theta $25.18^{\circ}$. All non-hydrogen atoms were refined anisotropically. The refinement showed a highly disordered tetrahydrofuran molecule, which could not be modelled satisfactorily. Therefore it was removed by using SQUEEZE routine of PLATON. A final refinement of 217 parameters has given $\mathrm{R} 1=0.0389, \mathrm{wR} 2=0.0653$ for the observed data. The crystal structure data are deposited to Cambridge Structural Database with CCDC number 1430855.

X-ray Crystallography: Crystals of 2a were grown in chloroform/acetone solution by slow evaporation method at room temperature. The crystals were adequately stable under ambient conditions, losing the crystalline nature for long exposure to air. The reported data set was collected by mounting the crystal with paratone oil in a quartz capillary at 150K. X-ray reflections were collected on the Rigaku Kappa 3 circle diffractometer with the AFC12 goniometer and enhanced sensitivity (HG) Saturn724+ CCD detector in the 4x4 bin mode using a monochromated Mo-K $\alpha$ radiation generated from the micro-focus sealed tube MicroMax-003 X-ray generator equipped with specially designed confocal multilayer optics. Data collection was performed using $\omega$-scans of $0.5^{\circ}$ steps at $150 \mathrm{~K}$. Cell determination, data collection and data reduction were performed with the help of Crystal Clear-SM Expert (version: 2.1-b24) software. ${ }^{4}$ The structure were solved by direct methods and refined by full-matrix least squares refinement methods based on $\mathrm{F}^{2}$, using SHELX-97 ${ }^{5}$ using the WinGX software suite. ${ }^{6}$ A total of 11491 reflections were measured out of which 2964 were independent and 2317 were observed [I $>2 \sigma(\mathrm{I})]$ for theta $25.46^{\circ}$. All non-hydrogen atoms were refined anisotropically. All hydrogen atoms were fixed geometrically with their $\mathrm{U}_{\text {iso }}$ values 1.2 times of the methyl and methylene carbons using a riding model. Important crystal and refinement parameters are given in. A final refinement of 209 parameters has given $R 1=0.0525$, wR2 $=0.0848$ for the observed data. The crystal structure data are deposited to Cambridge Structural Database with CCDC number 1412900.

Device Fabrication and Characterization: Prime grade p-doped silicon wafers (100) having a $300 \mathrm{~nm}$ thermally grown oxide layer (Montco Silicon) were used as device substrates. These were rinsed with water, methanol, and acetone before film deposition. Next, OTS-functionalized or PMMA (30 nm)-coated $\mathrm{Si} / \mathrm{SiO}_{2}$ substrates were obtained by dipping a 1M OTS solution in Xylene or is spin-coating a polymer solution (PMMA, $20 \mathrm{mg} \mathrm{mL}^{-1}$ in ethyl lactate, $9000 \mathrm{rpm}$ ), respectively. The dielectric film was dried at $110^{\circ} \mathrm{C}$ overnight in a vacuum oven $(<5 \mathrm{mTorr})$. The semiconductors were vacuum-deposited (growth rate $=$ 0.1-0.2 $\AA \mathrm{s} \mathrm{s}^{-1}$, thin-film thickness $=30 \mathrm{~nm}$, pressure $\sim 5 \times 10^{-6}$ Torr) onto substrates at different temperatures 
$\left(\mathrm{T}_{\mathrm{D}}=25^{\circ} \mathrm{C}\right.$ and $90{ }^{\circ} \mathrm{C}$ ). For TFT device fabrication, top-contact electrodes (500 $\AA$ ) were deposited by evaporating gold (pressure $\sim 10^{-6}$ Torr); channel dimensions were $100 \mu \mathrm{m}$ (L) by $1000 \mu \mathrm{m}$ (W). The capacitance of the insulator PMMA(30 nm)/SiO $2(300 \mathrm{~nm})$ is $10.0 \mathrm{nF} \mathrm{cm}{ }^{-2}$ whereas that of $\mathrm{OTS}-\mathrm{SiO}_{2}(300$ $\mathrm{nm}$ ) is $11.4 \mathrm{nF} \mathrm{cm}{ }^{-2}$. TFT device measurements were carried out in a customized vacuum probe station (pressure $\sim 10^{-6}$ Torr). Coaxial and/or triaxial shielding was incorporated into Signatone probes to minimize the noise level. TFT characterization was performed with a Keithley 6430 subfemtoammeter and a Keithley 2400 source meter, operated by a locally written LabView program and GPIB communication. Mobilities $(\mu)$ were calculated in the saturation regime by the standard relationship: $\mu_{\text {sat }}=\left(2 I_{\mathrm{DS}} L\right) /\left[W C_{\mathrm{i}}\left(V_{\mathrm{G}}-V_{\mathrm{T}}\right)^{2}\right]$, where $I_{D S}$ is the source-drain saturation current, $C_{i}$ is the gate dielectric capacitance (per area), $V_{G}$ is the gate voltage, and $\mathrm{V}_{\mathrm{T}}$ is the threshold voltage. The latter can be estimated as the $\mathrm{x}$ intercept of the linear section of the plot of $\mathrm{V}_{\mathrm{G}} \mathrm{VS}$. $\left(\mathrm{I}_{\mathrm{DS}}\right)^{1 / 2}$.

\section{Synthesis of Octabromoperylene-3,4,9,10-tetracarboxylicacid bisanhydride (1):}

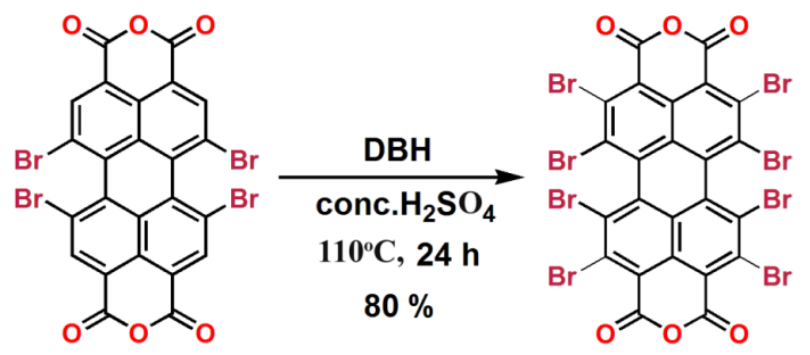

$2.68 \mathrm{~g}$ (10 mmol) of $\mathbf{B r}_{4}-\mathbf{P D A}^{7}$ and $20 \mathrm{~mL}$ concentrated sulfuric acid was stirred 5 min. to obtain a solution. Subsequently, $1.57 \mathrm{~g}(5 \mathrm{mmol})$ of DBH was added in portions over a period of $1 \mathrm{~h}$ and the RB flask was tightly closed by stop cork to avoid the escape of bromine from the reaction mixture. The resulting reddish brown solution was stirred at $110^{\circ} \mathrm{C}$ for $24 \mathrm{~h}$. After that the reddish brown reaction mixture was slowly poured into crushed ice. The resultant precipitate was filtered through a buchner funnel, washed with water 2-3 times and then with methanol. The solid was dried under vacuum to afford $\mathbf{1}$ as red solid. Yield $=80 \%$. Melting point: $>300{ }^{\circ} \mathrm{C} .{ }^{13} \mathrm{C}$ NMR (500 MHz, THF- $d_{8}$, TMS): $\delta=155.61,134.06$, 133.04, 131.27, 128.17, 127.92, 120.32. HR-ESI-MS: Calculated for $\mathrm{C}_{24} \mathrm{Br}_{8} \mathrm{O}_{6}(\mathrm{~m} / \mathrm{z})$ 1023.4852, found 1023.4882. FTIR (KBr pellet): $v=2924,2853,1784,1741,1632,1547,1364,1159,1054 \mathrm{~cm}^{-1}$. 


\section{Imidization of 1:}
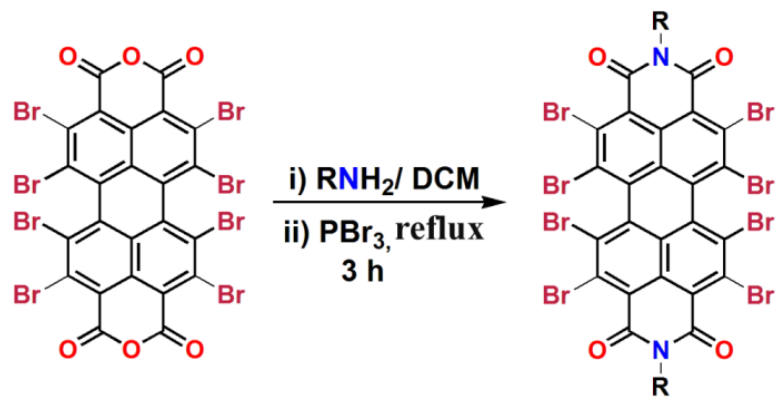

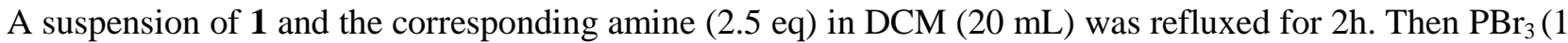
eq) was added to the reaction mixture and further refluxed for $1 \mathrm{~h}$. The reaction mixture was cooled to room temperature, poured into a separatory funnel and $100 \mathrm{~mL}$ of water was added. The aqueous layer was extracted twice with dichloromethane. The combined organic layer was dried over anhydrous $\mathrm{MgSO}_{4}$. The solvent was evaporated under reduced pressure and the crude product was purified by silica gel chromatography with hexane/dichloromethane (1:1) as eluent to afford 2a-e as red solids.

[Note: In the imidization reactions it is known that the alkyl amine/benzyl amine forms the intermediate carbamoyl derivative. $\mathrm{PBr}_{3}$ is added after $2 \mathrm{~h}$ to give sufficient time to form this intermediate. The intermediate then reacts with $\mathrm{PBr}_{3}$ to form the more reactive acyl bromide, which then undergo the imidization through cyclization.]

2a: Yield $=80 \% . \mathrm{R}_{f}=0.58\left(7: 3 \mathrm{CHCl}_{3} /\right.$ Hexane). Melting point $>30{ }^{\circ} \mathrm{C} .{ }^{1} \mathrm{H}$ NMR $\left(500 \mathrm{MHz}, \mathrm{CDCl}_{3}\right.$, TMS, 298 K): $\delta=4.26-4.20$ (m, 2H), 4.17- 4.11 (m, 2H), 1.72- 1.66 (m, 4H), 1.44- 1.39 (m, 4H), 0.94 (t, J $=7.5,6 \mathrm{H}) .{ }^{13} \mathrm{C}$ NMR $\left(125 \mathrm{MHz}, \mathrm{CDCl}_{3}, \mathrm{TMS}\right): \delta=160.10,133.54,132.78,132.05,127.60,125.83$, 121.94, 42.29, 30.06, 20.44, 13.86. MS (MALDI-TOF matrix: 1,8,9-Anthracenetriol): Calculated for $\mathrm{C}_{32} \mathrm{H}_{18} \mathrm{Br}_{8} \mathrm{~N}_{2} \mathrm{O}_{4}(\mathrm{~m} / \mathrm{z})$ 1133.73, found 1134.04). FTIR (KBr pellet): $v=2960,2930,2873,1707,1665,1551$, $1362 \mathrm{~cm}^{-1}$. Anal. Calcd. For $\mathrm{C}_{32} \mathrm{H}_{18} \mathrm{Br}_{8} \mathrm{~N}_{2} \mathrm{O}_{4}$ : C 33.90, H 1.60, N 2.47; Found: C 33.81, H 1.53, N 2.42.

2b: Yield $=70 \% . \mathrm{R}_{f}=0.58\left(7: 3 \mathrm{CHCl}_{3} /\right.$ Hexane $) \quad$ Melting point $>300^{\circ} \mathrm{C} .{ }^{1} \mathrm{H} \mathrm{NMR}\left(500 \mathrm{MHz}, \mathrm{CDCl}_{3}\right.$, TMS, $298 \mathrm{~K}): \delta=4.24-4.19$ (m, 2H), 4.14- 4.09 (m, 2H), 1.71- 1.66 (m, 4H), 1.38 -1.28 (m, 4H), 1.211.17 (m, 8H), 0.84 (t, J = 7.5, 6H). ${ }^{13} \mathrm{C}$ NMR (125 MHz, $\left.\mathrm{CDCl}_{3}, \mathrm{TMS}\right): \delta=160.08,133.53,132.75,132.03$, 127.60, 125.82, 121.94, 42.51, 31.54, 27.99, 26.85, 22.63, 14.09. MS (MALDI-TOF matrix: 1,8,9Anthracenetriol): Calculated for $\mathrm{C}_{36} \mathrm{H}_{26} \mathrm{Br}_{8} \mathrm{~N}_{2} \mathrm{O}_{4}(\mathrm{~m} / \mathrm{z})$ 1189.83, found 1190.16. FTIR (KBr pellet): $v=$ 2960, 2930, 2856, 1707, 1670, 1551, $1362 \mathrm{~cm}^{-1}$. Anal. Calcd. For $\mathrm{C}_{36} \mathrm{H}_{26} \mathrm{Br}_{8} \mathrm{~N}_{2} \mathrm{O}_{4}$ : C 36.34, H 2.20, N 2.35; Found: C 36.28, H 2.24, N 2.39

2c: Yield $=70 \% . \mathrm{R}_{f}=0.58\left(7: 3 \mathrm{CHCl}_{3} /\right.$ Hexane). Melting point $>300^{\circ} \mathrm{C} .{ }^{1} \mathrm{H} \mathrm{NMR}\left(500 \mathrm{MHz}, \mathrm{CDCl}_{3}\right.$, TMS, 298K): $\delta=4.23$ (m, 2H), 4.14 (m, 2H), 1.70 (m, 4H), 1.49 (m, 4H), $1.38(\mathrm{~m}, 4 \mathrm{H}), 1.31-1.22(\mathrm{~m}$, 12H), $0.81(\mathrm{t}, \mathrm{J}=7.5,6 \mathrm{H}) .{ }^{13} \mathrm{C} \mathrm{NMR}\left(125 \mathrm{MHz}, \mathrm{CDCl}_{3}, \mathrm{TMS}\right): \delta=160.10,133.53,132.76,132.04,127.60$, 125.81, 121.95, 42.52, 31.80, 29.71, 29.27, 28.06, 27.20, 22.71, 14.13. MS (MALDI-TOF matrix: 1,8,9Anthracenetriol): Calculated for $\mathrm{C}_{40} \mathrm{H}_{34} \mathrm{Br}_{8} \mathrm{~N}_{2} \mathrm{O}_{4}(\mathrm{~m} / \mathrm{z})$ 1245.94, found 1247.85. FTIR (KBr pellet): $v=$ 
2955, 2926, 2856, 1707, 1670, 1550, 1431, 1397, $1364 \mathrm{~cm}^{-1}$. Anal. Calcd. For $\mathrm{C}_{40} \mathrm{H}_{34} \mathrm{Br}_{8} \mathrm{~N}_{2} \mathrm{O}_{4}$ : C 38.56, $\mathrm{H}$ 2.75, N 2.25; Found: C 38.49, H 2.81, N 2.31.

2d:Yield $=20 \% . \mathrm{R}_{f}=0.58$ (7:3 $\mathrm{CHCl}_{3} /$ Hexane). Melting point $>300^{\circ} \mathrm{C} .{ }^{1} \mathrm{H}$ NMR $\left(500 \mathrm{MHz}, \mathrm{CDCl}_{3}\right.$, TMS, 298K): $\delta=4.95$ (m, 2H), 2.49 (m, 4H), 1.86-1.75 (m, 4H), 1.68 (m, 8H), 1.40 (m, 4H). ${ }^{13} \mathrm{C}$ NMR (125 MHz, $\mathrm{CDCl}_{3}$, TMS): $\delta=159.71,132.23,131.24,130.83,124.58,121.55,55.14,28.74,27.63,25.55$, 24.25. MS (MALDI-TOF matrix: 1,8,9-Anthracenetriol): Calculated for $\mathrm{C}_{36} \mathrm{H}_{22} \mathrm{Br}_{8} \mathrm{~N}_{2} \mathrm{O}_{4}$ (m/z) 1185.80, found 1186.34. FTIR (KBr pellet): $v=2926,2850,1711,1673,1548,1441,1373 \mathrm{~cm}^{-1}$. Anal. Calcd. For $\mathrm{C}_{36} \mathrm{H}_{22} \mathrm{Br}_{8} \mathrm{~N}_{2} \mathrm{O}_{4}$ : C 36.46, H 1.87, N 2.36; Found: C 36.51, H 1.84, N 2.40.

2e: Yield $=60 \%$. $\mathrm{R}_{f}=0.58\left(8: 2 \mathrm{CHCl}_{3} /\right.$ Hexane $)$ Melting point $>300^{\circ} \mathrm{C} .{ }^{1} \mathrm{H}$ NMR $\left(500 \mathrm{MHz}, \mathrm{CDCl}_{3}\right.$, TMS, $298 \mathrm{~K}): \delta=7.63$ (m, 4H), 7.53 (m, 4H), 5.51- 5.44 (m, 2H), 5.41- 5.36 (m, 2H). The poor solubility of compound $2 \mathbf{e}$ did not allow us to record ${ }^{13} \mathrm{C}$ NMR spectrum. MS (MALDI-TOF matrix: 1,8,9Anthracenetriol): Calculated for $\mathrm{C}_{40} \mathrm{H}_{12} \mathrm{Br}_{8} \mathrm{~F}_{6} \mathrm{~N}_{2} \mathrm{O}_{4}(\mathrm{~m} / \mathrm{z})$ 1337.76, found 1338.48. FTIR (KBr pellet): $v=$ 2966, 2929, 2858, 1707, 1670, 1547, 1431, $1389 \mathrm{~cm}^{-1}$. Anal. Calcd. For $\mathrm{C}_{40} \mathrm{H}_{12} \mathrm{Br}_{8} \mathrm{~F}_{6} \mathrm{~N}_{2} \mathrm{O}_{4}$ : C 35.91, $\mathrm{H}$ 0.90, N 2.09; Found: C 35.86, H 0.87, N 2.06.

\section{Synthesis of 5a-c:}

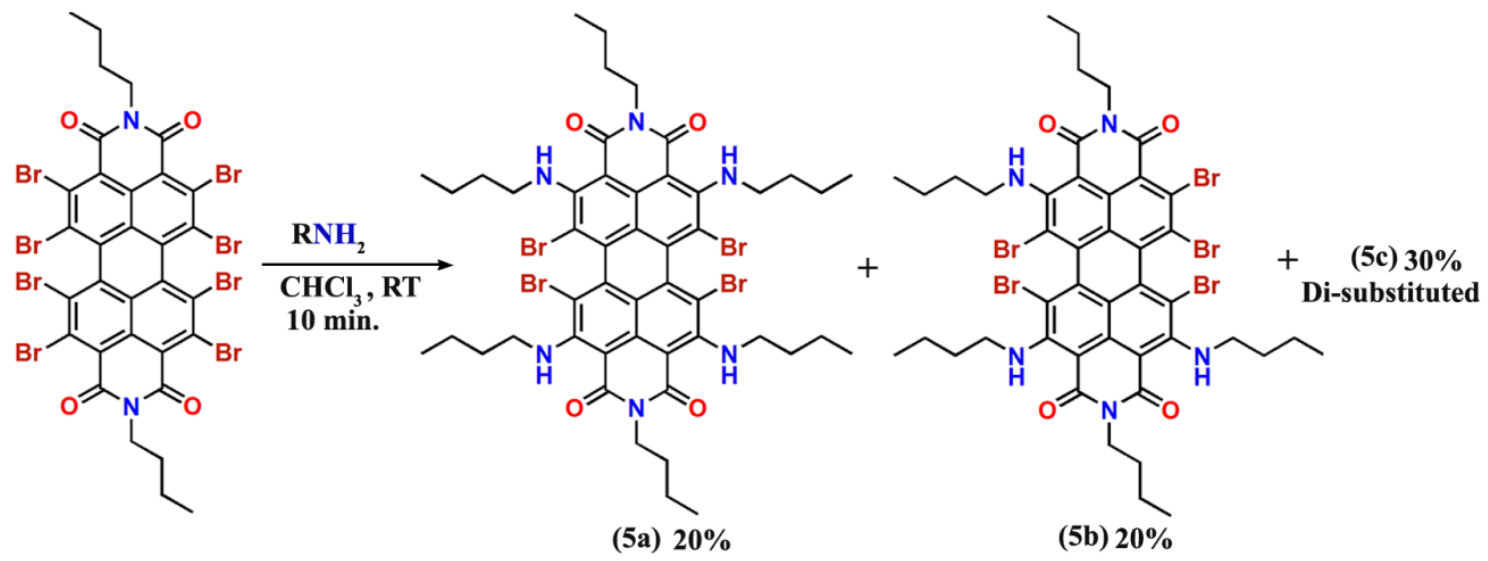

A suspension of $2 \mathbf{a}$ (200 mg, $0.18 \mathrm{mmol})$, n-butylamine (60 $\mu \mathrm{L}, 0.72 \mathrm{mmol})$ in chloroform (20 mL) was stirred at room temperature. The solution color changed from red to blue and the reaction was monitored by TLC. The solvent was evaporated under reduced pressure and the crude product was purified by silica gel chromatography with hexane/ethyl acetate (9:1) as eluent to afford $\mathbf{5 a}, \mathbf{5 b}$ and $\mathbf{5} \mathbf{c}$ as blue colored solids. 
5a: ${ }^{1} \mathrm{H}$ NMR (500 MHz, $\mathrm{CDCl}_{3}$, TMS, $298 \mathrm{~K}$ ): $\delta=9.89$ (s, 4H, NH), 4.17 (t, J = $7.5 \mathrm{~Hz}, 4 \mathrm{H}, \mathrm{NCH}_{2}$ ), 3.89 (m, 4H, $\mathrm{NHCH}_{2}$ ), 3.55 (m, 4H, $\mathrm{NHCH}_{2}$ ), 1.72 (m, 8H, $\left.\mathrm{CH}_{2}\right), 1.63$ (m, 4H, $\left.\mathrm{CH}_{2}\right), 1.42$ (m, 12H, $\left.\mathrm{CH}_{2}\right), 0.92$ $\left(\mathrm{m}, 18 \mathrm{H}, \mathrm{CH}_{3}\right) .{ }^{13} \mathrm{C} \mathrm{NMR}\left(125 \mathrm{MHz}, \mathrm{CDCl}_{3}, 298 \mathrm{~K}\right): \delta=163.80,153.55,135.75,128.17,116.14,111.05$, 101.88, 47.83, 39.25, 32.52, 29.14, 28.69, 19.42, 19.23, 12.95. MS (MALDI-TOF matrix: 1,8,9Anthracenetriol): Calculated for $\mathrm{C}_{48} \mathrm{H}_{58} \mathrm{Br}_{4} \mathrm{~N}_{6} \mathrm{O}_{4}(\mathrm{~m} / \mathrm{z})$ 1102.34, found 1103.01. FTIR (KBr pellet): $v=$ 3746, 2954, 2924, 2855, 1682, 1630, 1566, 1437, 1398, 1284, 1232, 1195, 1168, $1140 \mathrm{~cm}^{-1}$. Anal. Calcd. For $\mathrm{C}_{48} \mathrm{H}_{58} \mathrm{Br}_{4} \mathrm{~N}_{6} \mathrm{O}_{4}$ : C 52.29, H 5.30, N 7.62; Found: C 52.36, H 5.25, N 7.58.

5b: ${ }^{1} \mathrm{H}$ NMR (500 MHz, $\mathrm{CDCl}_{3}$, TMS, $\left.298 \mathrm{~K}\right): \delta=10.08$ (s, 1H, NH), 9.91 (s, 2H, NH), 4.16 (t, $J=7.5 \mathrm{~Hz}$, 4H, $\mathrm{NCH}_{2}$ ), 3.90 (m, 3H, $\left.\mathrm{NHCH}_{2}\right), 3.57\left(\mathrm{~m}, 3 \mathrm{H}, \mathrm{NHCH}_{2}\right), 1.67\left(\mathrm{~m}, 10 \mathrm{H}, \mathrm{CH}_{2}\right), 1.40\left(\mathrm{~m}, 10 \mathrm{H}, \mathrm{CH}_{2}\right), 0.88$ (m, 15H, $\left.\mathrm{CH}_{3}\right) .{ }^{13} \mathrm{C}$ NMR $\left(125 \mathrm{MHz}, \mathrm{CDCl}_{3}, 298 \mathrm{~K}\right): \delta=164.84,164.76,164.28,160.97,154.45,154.37$, 154.02, 137.29, 137.13 135.58, 134.21, 130.75, 129.17, 128.08, 126.02, 123.00, 119.54, 118.60, 117.08, 111.92, 111.87, 103.43, 102.45, 100.99, 49.13, 48.91, 48.64, 41.23, 40.34, 33.52, 33.36, 31.94, 30.15, 30.08, 29.71, 29.38, 22.71, 20.49, 20.25, 20.20, 14.14, 13.98. MS (MALDI-TOF matrix: 1,8,9Anthracenetriol): Calculated for $\mathrm{C}_{44} \mathrm{H}_{48} \mathrm{Br}_{5} \mathrm{~N}_{5} \mathrm{O}_{4}(\mathrm{~m} / \mathrm{z})$ 1110.02, found 1109.74. FTIR (KBr pellet): $v=$ 3746, 2958, 2932, 2871, 1641, 1557, 1455, 1282, 1195, 1169, $1128 \mathrm{~cm}^{-1}$. Anal. Calcd. For $\mathrm{C}_{44} \mathrm{H}_{48} \mathrm{Br}_{5} \mathrm{~N}_{5} \mathrm{O}_{4}$ : C 47.59, H 4.30, N 6.30; Found: C 47.53, H 4.27, N 6.36.

5c (disubstituted): ${ }^{1} \mathrm{H}$ NMR (500 MHz, $\left.\mathrm{CDCl}_{3}, \mathrm{TMS}, 298 \mathrm{~K}\right): \delta=10.10$ (s, 2H, NH), 4.17 (m, 4H, $\mathrm{NCH}_{2}$ ), 3.99 (m, 2H, $\mathrm{NHCH}_{2}$ ), 3.56 (m, 2H, $\mathrm{NHCH}_{2}$ ), 1.76 (m, 4H, $\mathrm{CH}_{2}$ ), 1.67 (m, 4H, $\left.\mathrm{CH}_{2}\right), 1.43$ (m, 8H, $\mathrm{CH}_{2}$ ), 0.93 (m, 12H, $\left.\mathrm{CH}_{3}\right) .{ }^{13} \mathrm{C}$ NMR (125 MHz, $\left.\mathrm{CDCl}_{3}, 298 \mathrm{~K}\right): \delta=163.33,159.78,152.95,136.53,131.93$, 129.73, 126.99, 125.06, 122.18, 119.10, 117.70, 99.69, 47.90, 40.26, 32.29, 29.01, 28.68, 19.45, 19.14, 12.89. MS (MALDI-TOF matrix: 1,8,9-Anthracenetriol): Calculated for $\mathrm{C}_{40} \mathrm{H}_{38} \mathrm{Br}_{6} \mathrm{~N}_{4} \mathrm{O}_{4}(\mathrm{~m} / \mathrm{z})$ 1118.50, found 1119.05. FTIR (KBr pellet): $v=3746,2956,2929,2869,1647,1564,1457,1284,1194,1163,1115$ $\mathrm{cm}^{-1}$. Anal. Calcd. For $\mathrm{C}_{40} \mathrm{H}_{38} \mathrm{Br}_{6} \mathrm{~N}_{4} \mathrm{O}_{4}$ : C 42.97, H 3.43, N 5.01; Found: C 42.92, H 3.39, N 5.08.

Synthesis of the radical anion of 2a: We prepared the 2a radical anion by addition of TBACN in dry $\mathrm{CH}_{3} \mathrm{CN}$. The 2a radical anion was isolated from the solution as a TBA salt under Ar. The isolated solid was then recrystallized from DCM/hexane mixtures. The ESI mass, UV- visible spectrum of the radical anion and the EPR spectrum of the isolated solid was subsequently recorded.

Synthesis of $\mathbf{3}$ and 4: $\mathbf{3}$ and $\mathbf{4}$ were synthesized according to the literature procedures. ${ }^{7,8}$ 
Table S1: Comparison of the selected bond length, angle and torsion angle of the crystal and geometry of the optimized structures of $\mathbf{1}$ and $\mathbf{2 a}$.

\begin{tabular}{|c|c|c|c|c|}
\hline Atoms & $\begin{array}{l}\text { Selected Bond } \\
\text { length }[\AA] \\
\text { Crystal Data }(\mathbf{1}) \\
\end{array}$ & $\begin{array}{c}\text { Selected Bond } \\
\text { length }[\AA] \text { Crystal } \\
\text { Data }(\mathbf{2 a}) \\
\end{array}$ & $\begin{array}{c}\text { Selected Bond } \\
\text { length }[\AA] \text { B3LYP } \\
\text { 6-311G (1) } \\
\end{array}$ & $\begin{array}{c}\text { Selected Bond } \\
\text { length }[\AA] \text { B3LYP } \\
\text { 6-311G (2a) } \\
\end{array}$ \\
\hline $\mathrm{C} 1-\mathrm{O} 1, \mathrm{C} 1{ }^{\prime}-\mathrm{O} 1^{\prime}$ & $1.173 \AA$ & $1.205 \AA$ & $1.218 \AA$ & $1.243 \AA$ \\
\hline $\mathrm{C} 10-\mathrm{O} 2, \mathrm{C} 10^{\prime}-\mathrm{O} 2{ }^{\prime}$ & $1.197 \AA$ & $1.217 \AA$ & $1.218 \AA$ & $1.243 \AA$ \\
\hline $\mathrm{C} 1-\mathrm{C} 2, \mathrm{C} 1{ }^{\prime}-\mathrm{C} 2{ }^{\prime}$, & $1.492 \AA$ & $1.499 \AA$ & $1.476 \AA$ & $1.482 \AA$ \\
\hline $\mathrm{C} 2-\mathrm{C} 3, \mathrm{C} 2{ }^{\prime}-\mathrm{C} 3^{\prime}$ & $1.391 \AA$ & $1.395 \AA$ & $1.397 \AA$ & $1.399 \AA$ \\
\hline $\mathrm{C} 3-\mathrm{C} 4, \mathrm{C} 3^{\prime}-\mathrm{C} 4{ }^{\prime}$ & $1.415 \AA$ & $1.430 \AA$ & $1.416 \AA$ & $1.417 \AA$ \\
\hline $\mathrm{C} 4-\mathrm{C} 5, \mathrm{C} 4^{\prime}-\mathrm{C} 5^{\prime}$ & $1.380 \AA$ & $1.388 \AA$ & $1.396 \AA$ & $1.394 \AA$ \\
\hline $\mathrm{C} 5-\mathrm{C} 12, \mathrm{C} 5^{\prime}-\mathrm{C} 12^{\prime}$ & $1.422 \AA$ & $1.423 \AA$ & $1.426 \AA$ & $1.425 \AA$ \\
\hline C12 - C6, C12' - C6' & $1.429 \AA$ & $1.414 \AA$ & $1.426 \AA$ & $1.425 \AA$ \\
\hline $\mathrm{C} 6-\mathrm{C} 7, \mathrm{C} 6^{\prime}-\mathrm{C} 7^{\prime}$ & $1.395 \AA$ & $1.380 \AA$ & $1.396 \AA$ & $1.394 \AA$ \\
\hline $\mathrm{C} 7-\mathrm{C} 8, \mathrm{C} 7^{\prime}-\mathrm{C} 8{ }^{\prime}$ & $1.411 \AA$ & $1.417 \AA$ & $1.416 \AA$ & $1.417 \AA$ \\
\hline $\mathrm{C} 8$ - C9, C8' - C9' & $1.369 \AA$ & $1.392 \AA$ & $1.397 \AA$ & $1.399 \AA$ \\
\hline $\mathrm{C} 9-\mathrm{C} 10, \mathrm{C} 9^{\prime}-\mathrm{C} 10^{\prime}$ & $1.488 \AA$ & $1.479 \AA$ & $1.476 \AA$ & $1.482 \AA$ \\
\hline Atom & \multicolumn{4}{|c|}{ Selected bond angle } \\
\hline $\mathrm{C} 4-\mathrm{C} 5-\mathrm{C} 5$ & $126.33^{\circ}$ & $126.44^{\circ}$ & $126.01^{\circ}$ & $126.28^{\circ}$ \\
\hline C7-C6-C6' & $125.59^{\circ}$ & $124.84^{\circ}$ & $126.01^{\circ}$ & $126.29^{\circ}$ \\
\hline Atom & \multicolumn{4}{|c|}{ Selected torsion angle } \\
\hline C4 - C5 - C5' - C4' & $-34.90^{\circ}$ & $-39.78^{\circ}$ & $41.74^{\circ}$ & $42.63^{\circ}$ \\
\hline C7 - C6 - C6' - C7' & $-39.35^{\circ}$ & $-42.71^{\circ}$ & $41.75^{\circ}$ & $42.63^{\circ}$ \\
\hline C7 - C6 - C6' - C12' & $143.05^{\circ}$ & $140.37^{\circ}$ & $-140.81^{\circ}$ & $-140.05^{\circ}$ \\
\hline C4 - C5 - C5'-C12' & $143.89^{\circ}$ & $142.46^{\circ}$ & $-140.81^{\circ}$ & $-139.90^{\circ}$ \\
\hline
\end{tabular}

Table S2: C-Br---O, C-Br--- $\pi$ and $\mathrm{Br}---\mathrm{Br}$ non-bonding interactions in crystals of $\mathbf{1}$ and $\mathbf{2 a}$.

\begin{tabular}{|c|c|c|c|c|c|c|c|c|}
\hline \multirow[t]{2}{*}{ Mol. } & \multicolumn{3}{|c|}{$\begin{array}{l}\text { C-Br---O Non-Bonding } \\
\text { Interaction }\end{array}$} & \multicolumn{5}{|c|}{$\mathrm{Br}---\pi$ and $\mathrm{Br}---\mathrm{Br}$ Non-Bonding Interaction } \\
\hline & $\begin{array}{c}\text { Br---0 } \\
(\AA)\end{array}$ & $\begin{array}{c}\text { C---0 } \\
(\AA \AA)\end{array}$ & $\begin{array}{c}\angle \mathrm{C}-\mathrm{Br}---\mathrm{O} \\
\left({ }^{\circ}\right)\end{array}$ & $\begin{array}{c}\text { Br--- } \boldsymbol{\pi} \\
(\AA)\end{array}$ & $\begin{array}{c}\angle \mathrm{C}-\mathrm{Br}-\pi \\
\left(^{\circ}\right)\end{array}$ & $\begin{array}{c}\text { Br---Br } \\
(\AA)\end{array}$ & $\begin{array}{c}\angle \mathrm{C}-\mathrm{Br}---\mathrm{Br}{ }^{\prime} \\
\left({ }^{\circ}\right)\end{array}$ & $\begin{array}{c}\angle \mathrm{C}-\mathrm{Br}{ }^{\prime}---\mathrm{Br} \\
\left({ }^{\circ}\right)\end{array}$ \\
\hline \multirow[t]{2}{*}{1} & 2.936 & 4.795 & 167.54 & - & - & 3.448 & 161 & 93 \\
\hline & 3.223 & 4.529 & 122.26 & - & - & - & - & - \\
\hline \multirow[t]{2}{*}{$2 \mathbf{2 a}$} & 2.794 & 4.673 & 174.04 & 3.404 & 144.40 & - & - & - \\
\hline & 3.250 & 4.709 & 130.84 & - & - & - & - & - \\
\hline
\end{tabular}

${ }^{*}$ In type $\mathrm{I}$, the centroid was determined using atoms $\mathrm{C}_{2}, \mathrm{C}_{3}$ and $\mathrm{C}_{4}$ and in type $\mathrm{II}_{5}, \mathrm{C}_{11}$ and $\mathrm{C}_{12}$. 
Table S3A: Optical properties and reduction potentials of 1-4

\begin{tabular}{|c|c|c|c|c|c|c|c|}
\hline Entry & \multicolumn{3}{|c|}{$\lambda_{\max }^{\mathrm{abs}}\left(\mathrm{cm}^{-1}\right) /(\mathrm{nm})$} & \multirow{2}{*}{$\begin{array}{c}\lambda_{\max }^{\mathrm{emi}}\left(\mathrm{cm}^{-1}\right) \\
/(\mathrm{nm}) /(\phi)^{\mathrm{a}} \\
16583.75 \\
(603) /(0.25)\end{array}$} & \multirow{2}{*}{$\begin{array}{c}\mathrm{E}^{1}{ }_{\text {red }} / \mathrm{V} \\
\mathrm{CV} / \mathrm{DPV} \\
+0.04 /+0.06\end{array}$} & \multirow{2}{*}{$\begin{array}{c}\mathrm{E}^{2} \text { red } / \mathrm{V} \\
\mathrm{CV} / \mathrm{DPV} \\
-0.15 /-0.28\end{array}$} & \multirow{2}{*}{$\begin{array}{l}\text { LUMO } \\
(\mathrm{eV}) \\
\text { exp. } \\
-4.46\end{array}$} \\
\hline 1 & - & $\begin{array}{c}21598.27 \\
(463)\end{array}$ & $\begin{array}{c}1897533 \\
(527)\end{array}$ & & & & \\
\hline $2 a$ & - & $\begin{array}{c}21367.52 \\
(468) \\
\end{array}$ & $\begin{array}{c}18656.52 \\
(536) \\
\end{array}$ & $\begin{array}{c}16778.52 \\
(596) /(0.33)\end{array}$ & $-0.17 /-0.20$ & $-0.34 /-0.37$ & -4.23 \\
\hline $2 b$ & - & $\begin{array}{c}21413.28 \\
(467)\end{array}$ & $\begin{array}{c}18726.59 \\
(534)\end{array}$ & $\begin{array}{c}16835.02 \\
(594) /(0.33)\end{array}$ & $-0.18 /-0.16$ & $-0.35 /-0.33$ & -4.22 \\
\hline $2 c$ & - & $\begin{array}{c}21367.52 \\
(468)\end{array}$ & $\begin{array}{c}18691.59 \\
(535)\end{array}$ & $\begin{array}{c}16949.15 \\
(590) /(0.33)\end{array}$ & $-0.20 /-0.17$ & $-0.41 /-0.37$ & -4.20 \\
\hline $2 d$ & - & $\begin{array}{c}21367.52 \\
(468)\end{array}$ & $\begin{array}{c}18691.59 \\
(535)\end{array}$ & $\begin{array}{c}17241.38 \\
(580) /(0.33)\end{array}$ & $-0.17 /-0.13$ & $-0.24 /-0.30$ & -4.26 \\
\hline $2 e$ & - & $\begin{array}{c}21276.60 \\
(470)\end{array}$ & $\begin{array}{c}18621.37 \\
(537)\end{array}$ & $\begin{array}{c}17331.02 \\
(577) /(0.31)\end{array}$ & $-0.11 /-0.09$ & $-0.30 /-0.28$ & -4.31 \\
\hline 3 & $\begin{array}{c}22222.22 \\
(450) \\
\end{array}$ & $\begin{array}{c}20533.38 \\
(487) \\
\end{array}$ & $\begin{array}{c}19157.09 \\
(522) \\
\end{array}$ & $\begin{array}{c}18214.94 \\
(549) /(0.95)\end{array}$ & $-0.55 /-0.53$ & $-0.74 /-0.72$ & -3.86 \\
\hline 4 & $\begin{array}{c}22883.30 \\
(437)\end{array}$ & $\begin{array}{c}20283.98 \\
(493)\end{array}$ & $\begin{array}{c}19011.41 \\
(526)\end{array}$ & $\begin{array}{c}17761.99 \\
(563) /(0.46)\end{array}$ & $-0.43 /-0.40$ & $-0.62 /-0.59$ & -3.99 \\
\hline
\end{tabular}

UV-Vis and fluorescence spectra of 1, 2a-e, 3 and 4 in $\mathrm{CH}_{2} \mathrm{Cl}_{2},\left(\lambda_{\mathrm{ex}} \sim 500 \mathrm{~nm}\right), \mathrm{LUMO}=\left[-4.4-\mathrm{E}_{1}{ }^{\mathrm{R}}\right] \mathrm{eV}$ against vacuum, LUMO was estimated with respect to the DPV, SCE, $0.1 \mathrm{M} \mathrm{Bu}_{4} \mathrm{NPF}_{6}, 298 \mathrm{~K}$.

${ }^{\mathrm{a}} \Phi=$ quantum yield, reference $=$ fluorescein $(\varphi=0.92)$ in $\left(0.1 \mathrm{~N} \mathrm{NaOH}, \eta_{R}=1.33\right)$.

$\Phi=\Phi_{R}\left[\frac{\mathrm{I}}{\mathrm{I}_{R}}\right]\left[\frac{\mathrm{A}_{R}}{\mathrm{~A}}\right]\left[\frac{\eta}{\eta_{R}}\right]^{2}$

$\Phi_{R}=$ reference quantum yield, $\mathbf{I}=$ integrated fluorescence intensity, $\mathbf{A}=$ Absorbance, $\mathbf{\eta}=$ refractive index ( $R$ for references). The fluorescence and absorbance spectra of the compounds were recorded in dichloromethane and refractive index $(\mathbf{\eta})$ of DCM $=1.42$ 
Table S3B: Optical properties of 5a-c

\begin{tabular}{|c|c|c|c|}
\hline Entry & $\lambda_{\max }^{\text {abs }}\left(\mathrm{cm}^{-1}\right) /(\mathrm{nm})$ & $\lambda_{\max }^{\text {emi }}\left(\mathrm{cm}^{-1}\right) /(\mathrm{nm})$ & Quantum yield $(\varphi)$ \\
\hline $\mathbf{5 a}$ & $17391.30 / 575$ & $23255.81 / 430$ & 0.16 \\
\hline $\mathbf{5 b}$ & $17574.69 / 569$ & $23148.15 / 432$ & 0.05 \\
\hline $\mathbf{5 c}$ & $16750.42 / 597$ & $23282.89 / 429.5$ & 0.03 \\
\hline
\end{tabular}

Table S4: Electron mobilities of the organic semiconductors (OSC) on OTS functionalized or PMMA-coated n-doped $\mathrm{Si} / \mathrm{SiO}_{2}$ substrates under $\mathrm{RT}$ and $90^{\circ} \mathrm{C}$ :

\begin{tabular}{|c|c|c|c|c|c|c|}
\hline & \multirow{2}{*}{ OSC } & \multirow[b]{2}{*}{$\begin{array}{l}\text { Von } \\
\text { (V) }\end{array}$} & \multicolumn{4}{|c|}{$\mu_{\mathrm{s}}\left(\mathrm{cm}^{2} / \mathrm{Vs}\right)$} \\
\hline & & & SiO2-PMMA & SiO2-OTS & $\begin{array}{c}\text { SiO2-PMMA } \\
\left(90^{\circ} \mathrm{C}\right)\end{array}$ & $\begin{array}{l}\text { SiO2-OTS } \\
\left(90^{\circ} \mathrm{C}\right)\end{array}$ \\
\hline $2 \mathrm{a}$ & $\mathrm{R}=\mathrm{C}_{4} \mathrm{H}_{9}$ & $9 \sim 45$ & $5.48 \mathrm{E}-04$ & $6.34 \mathrm{E}-04$ & $<5.0 \mathrm{E}-05$ & $1.72 \mathrm{E}-03$ \\
\hline $2 b$ & $\mathrm{R}=\mathrm{C}_{6} \mathrm{H}_{13}$ & $41 \sim 48$ & $3.60 \mathrm{E}-04$ & N/A & N/A & $N / A$ \\
\hline $2 \mathrm{c}$ & $\mathrm{R}=\mathrm{C}_{8} \mathrm{H}_{17}$ & $25 \sim 27$ & $<1.0 \mathrm{E}-04$ & N/A & N/A & N/A \\
\hline $\begin{array}{l}\mathbf{2 e} \\
\mathrm{R}=\end{array}$ & $\left.\mathrm{I}_{2}\right) \mathrm{p}-\mathrm{C}_{5} \mathrm{H}_{4} \mathrm{CF}_{3}$ & $19 \sim 22$ & $<5.0 \mathrm{E}-05$ & $<1.5 \mathrm{E}-04$ & N/A & N/A \\
\hline
\end{tabular}

* N/A: Not active 


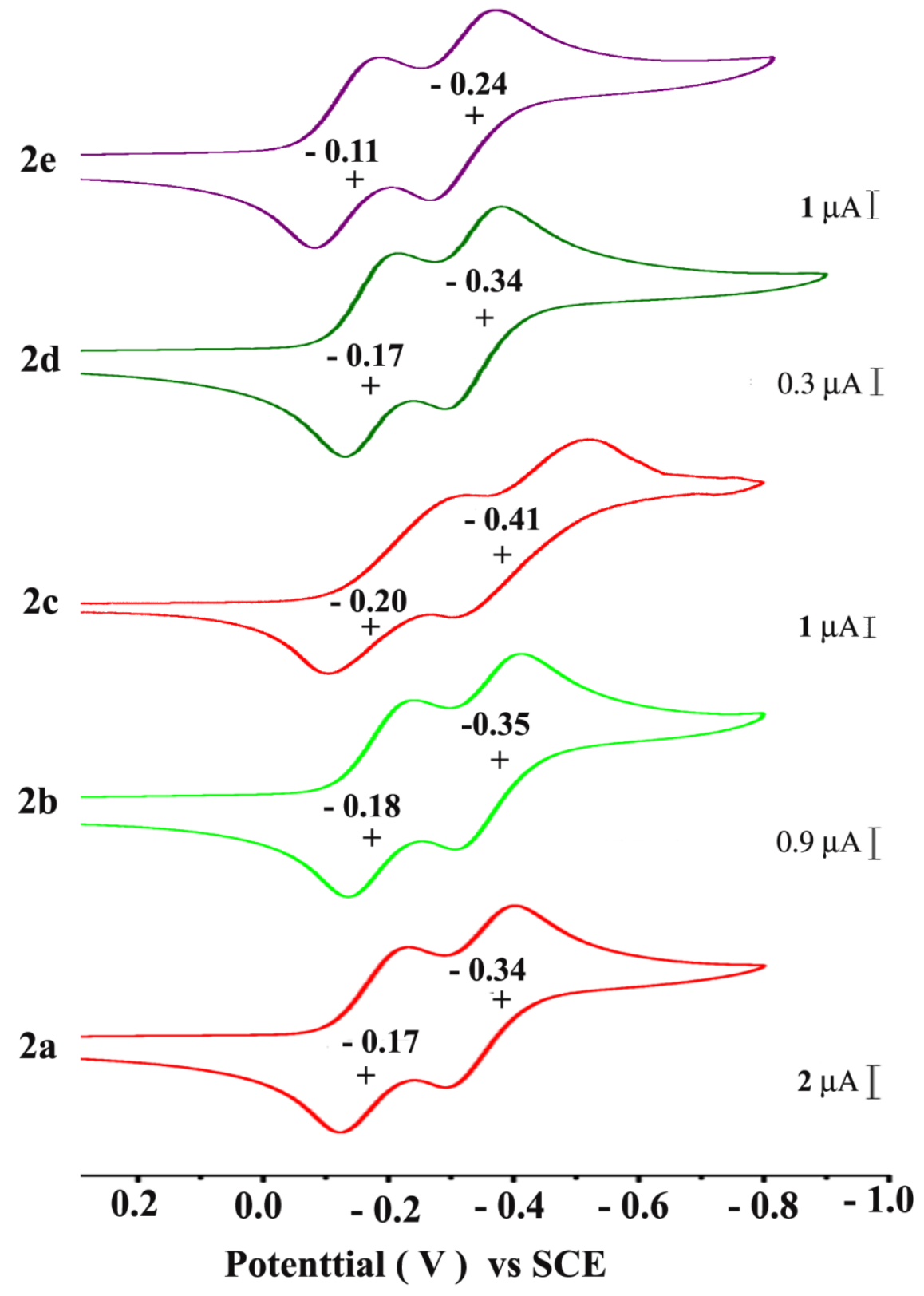

Figure S1: CV results of 2a-e $\left(5 \times 10^{-4} \mathrm{M}\right)$ in degassed DCM at $298 \mathrm{~K}$. 


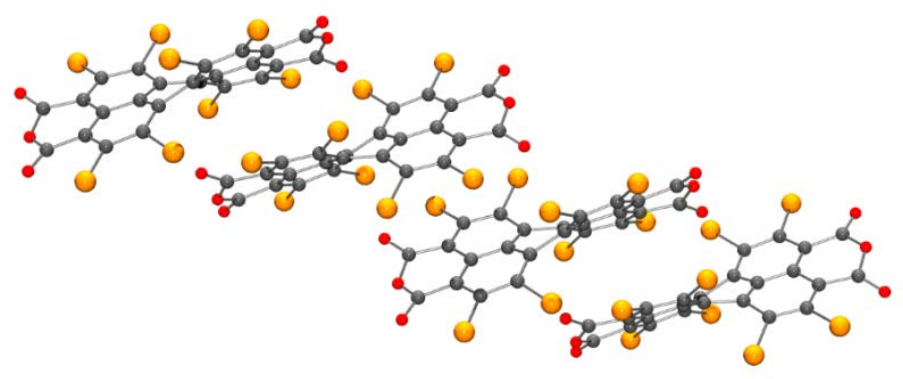

Figure S2: A POV-ray diagram of the self-assembly network of $\mathbf{1}$ along the $\mathbf{b}$ axis.

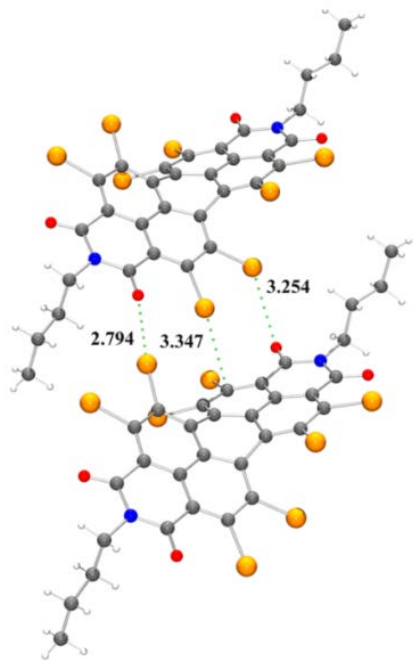

Figure S3: Non-bonding interactions of $\mathbf{2 a}$ showing the Br---O interactions.

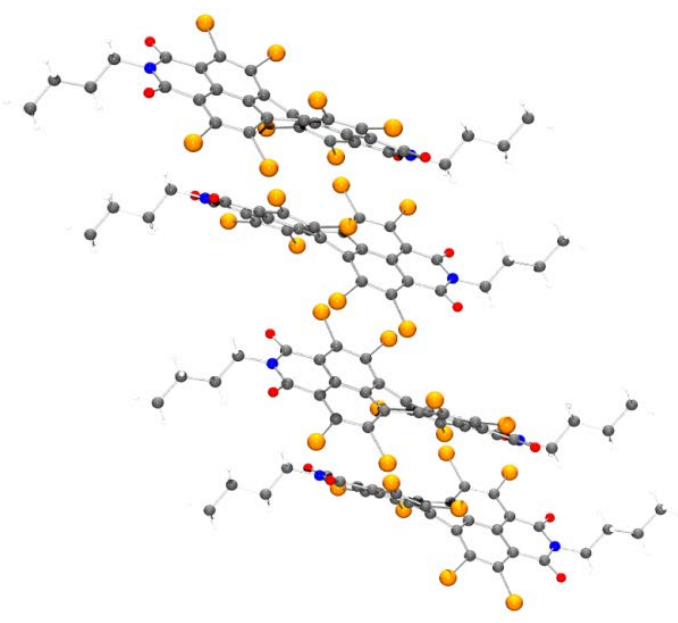

Figure S4: A POV-ray diagram of the self-assembly network of $2 \mathbf{a}$ along the a axis. 

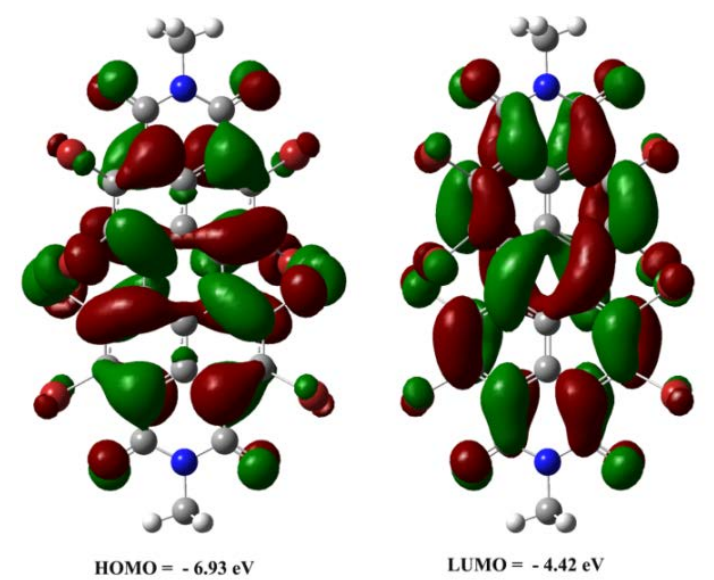

Figure S5: Electronic density contours of the HOMO (left) and LUMO (right) of 2a. The molecular structure was optimized using B3LYP/6-311G(d,p).

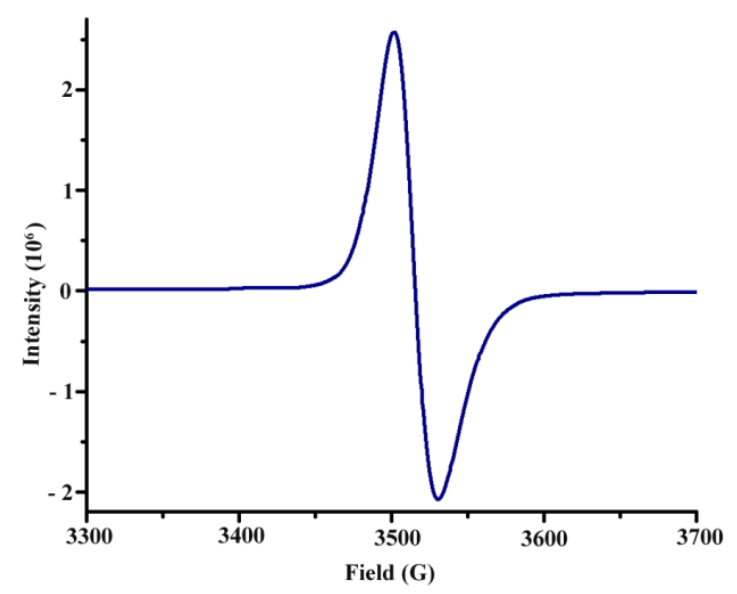

Figure S6: ESR spectrum of $\mathbf{2 a}^{\mathbf{*}^{-}}$in the solid state at $298 \mathrm{~K}$ [g $\left.=2.004\right]$. 


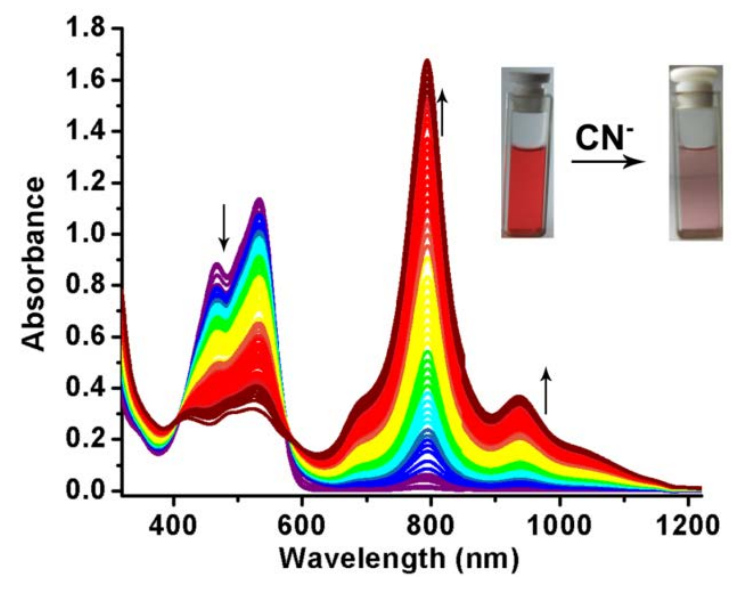

Figure S7: UV-vis-NIR spectra showing the response of $2 \mathbf{a}\left(5 \times 10^{-5} \mathrm{M}\right)$ in $\mathrm{CH}_{3} \mathrm{CN}$ with gradual addition of $\mathrm{CN}^{-}(0.0$ - 1.0 equiv.) and formation of 2a- under ambient condition.

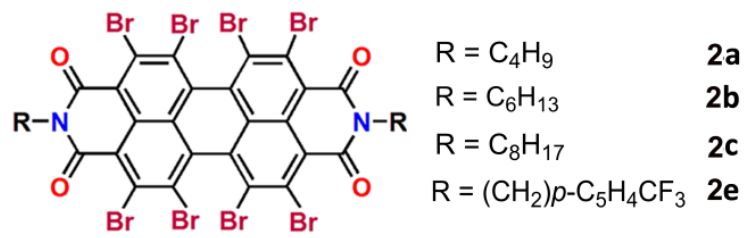

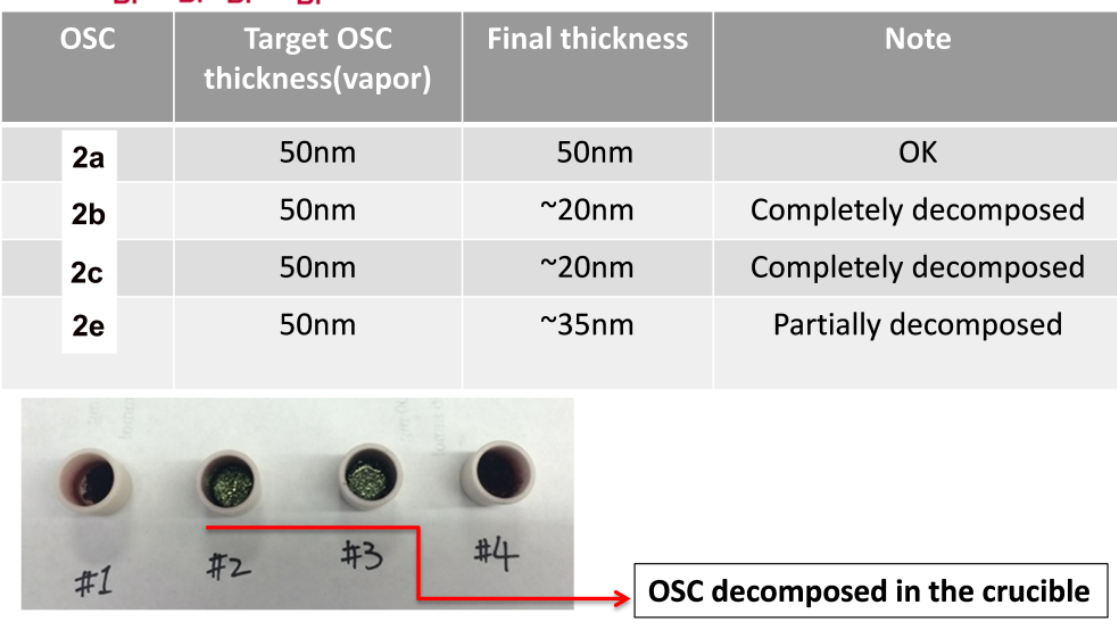

Figure S8: Top: Molecular structures, Middle: Characteristics of the deposited thin films Bottom: Photographs of the residues in the crucibles. 


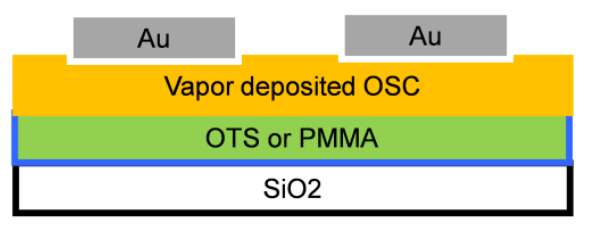

Vds $=-100 \mathrm{~V}$; Device test in Vacuum
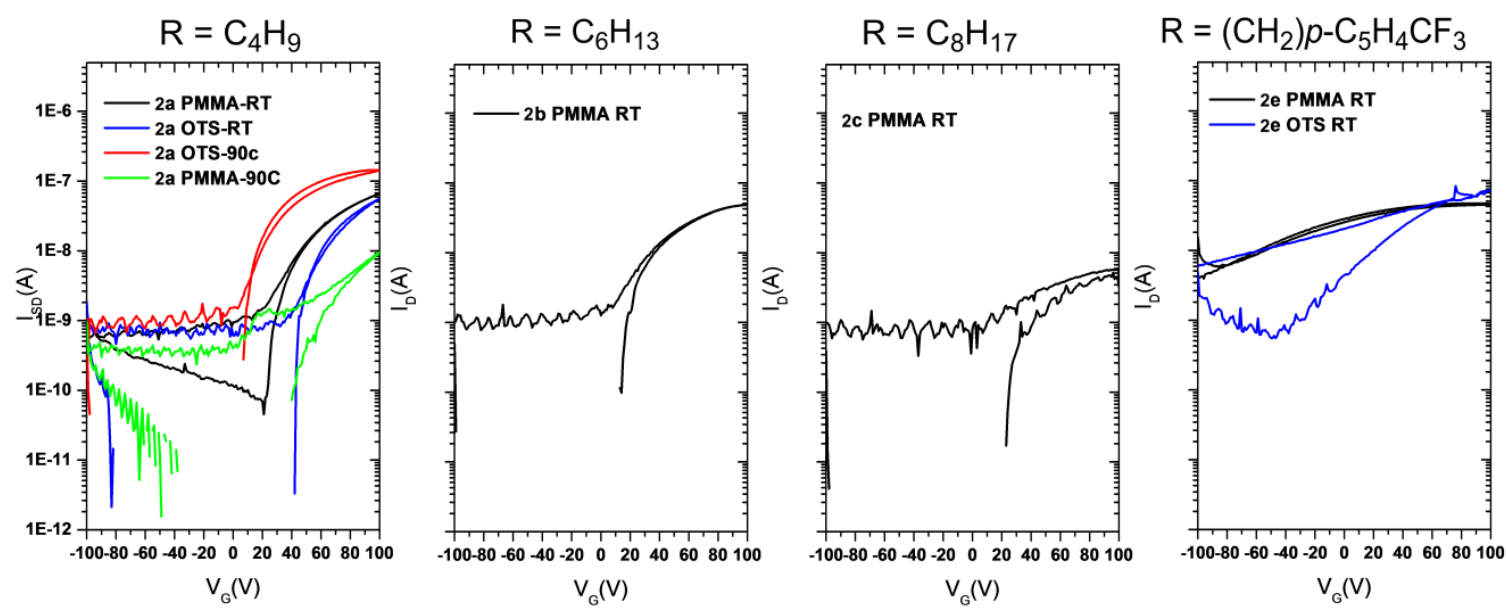

Figure S9: Top: Architecture of the bottom-gate top-contact OTFT. Bottom: I-V plots of $\mathbf{2 a}, \mathbf{2 b}$, 2c and 2e (from left to right) on OTS functionalized or PMMA-coated n-doped $\mathrm{Si}^{-} \mathrm{SiO}_{2}$ substrates under RT or $90^{\circ} \mathrm{C}$.

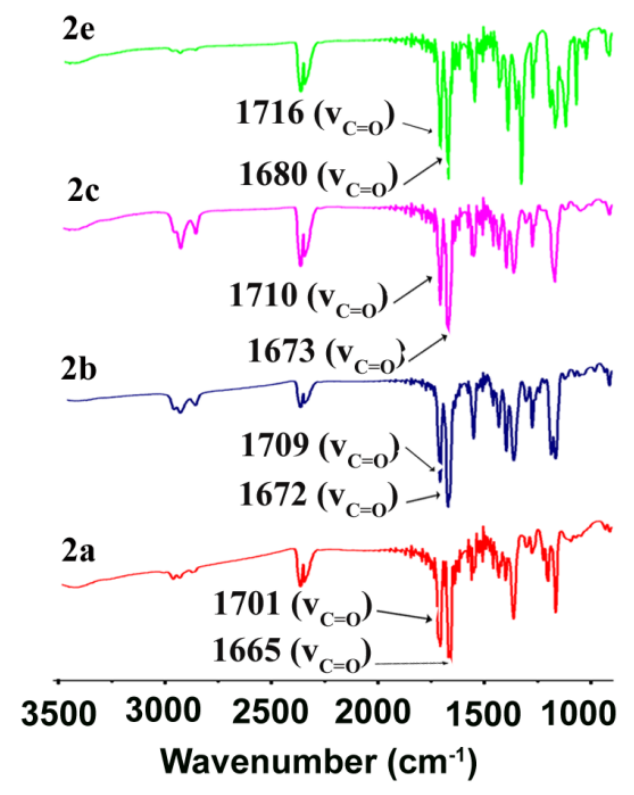

Figure S10: FTIR spectra of 2a, 2b, 2c and 2e. 


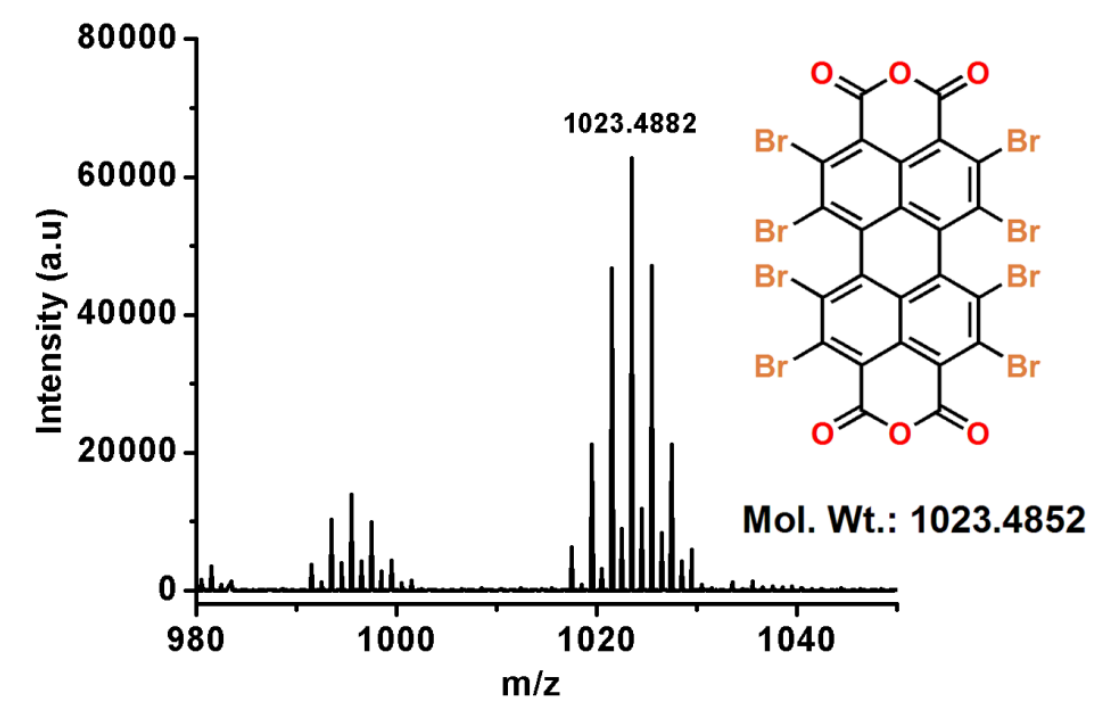

Figure S11: HR-ESI-MS spectrum of 1.

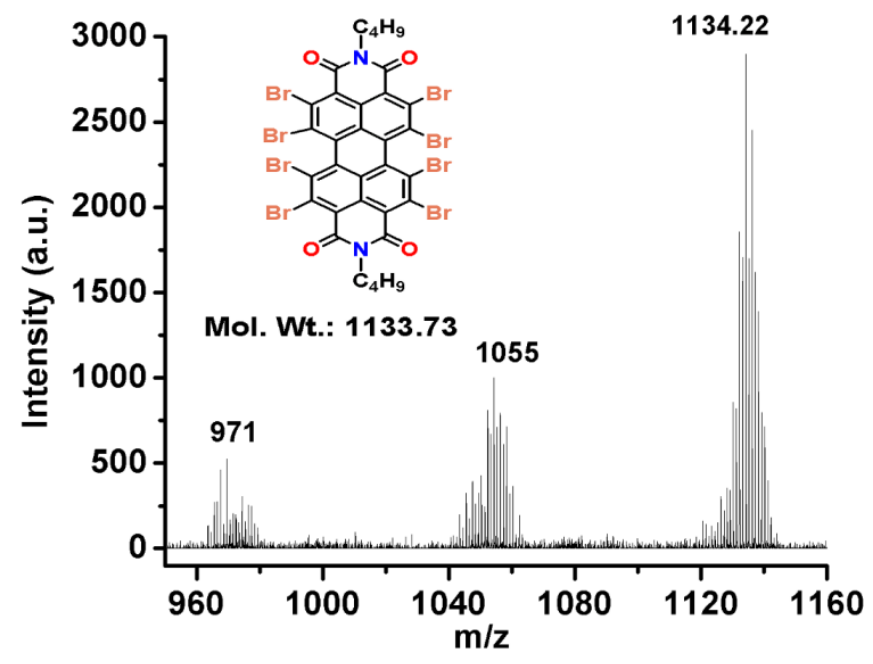

Figure S12: MALDI-TOF mass spectrum of 2a. 


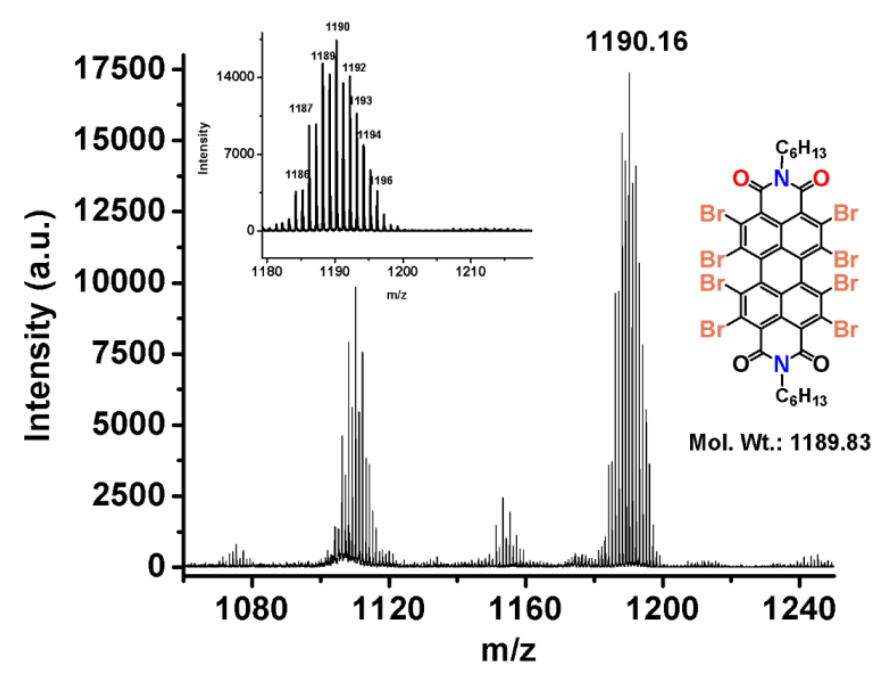

Figure S13: MALDI-TOF mass spectrum of $\mathbf{2 b .}$

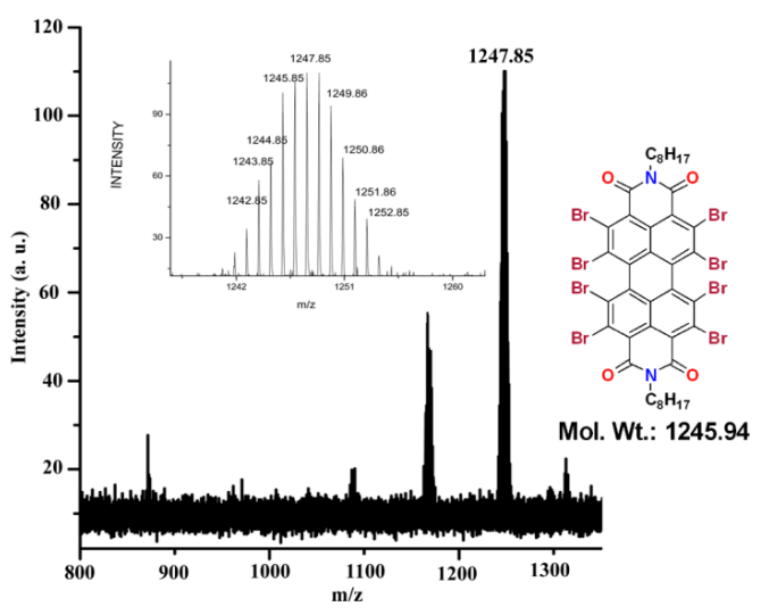

Figure S14: MALDI-TOF mass spectrum of 2c.

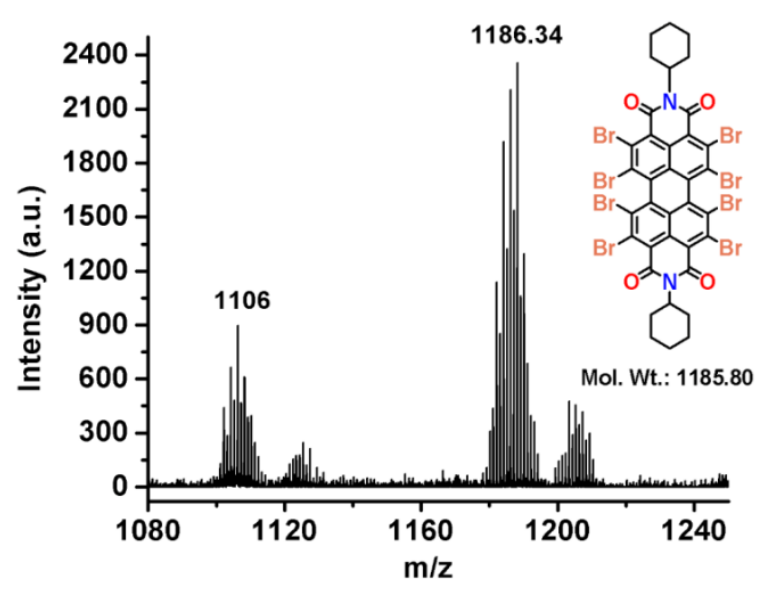

Figure S15: MALDI-TOF mass spectrum of 2d. 


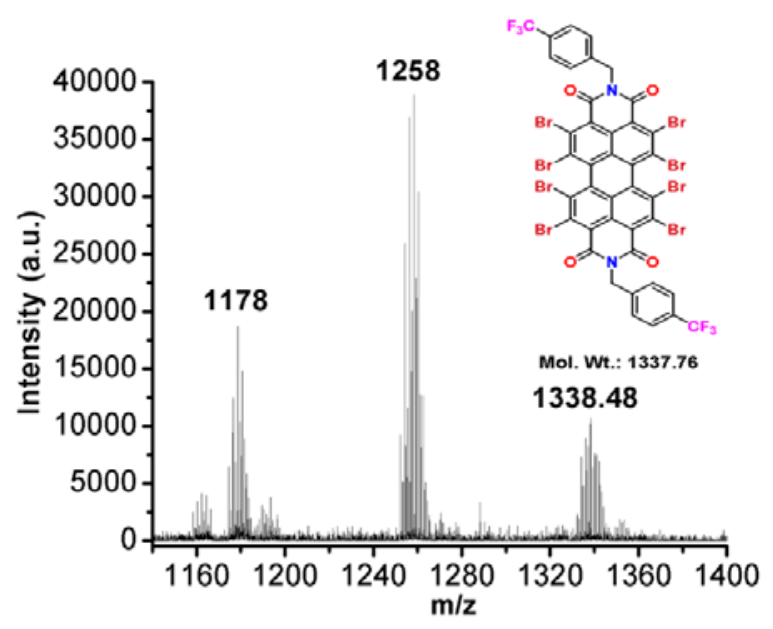

Figure S16: MALDI-TOF mass spectrum of $\mathbf{2 e .}$

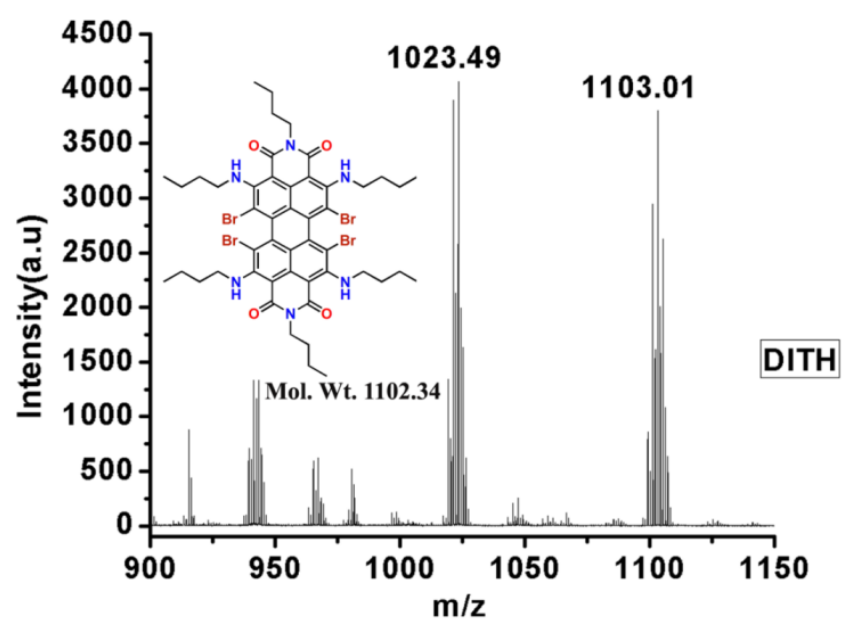

Figure S17: MALDI-TOF mass spectrum of $\mathbf{5 a .}$ 


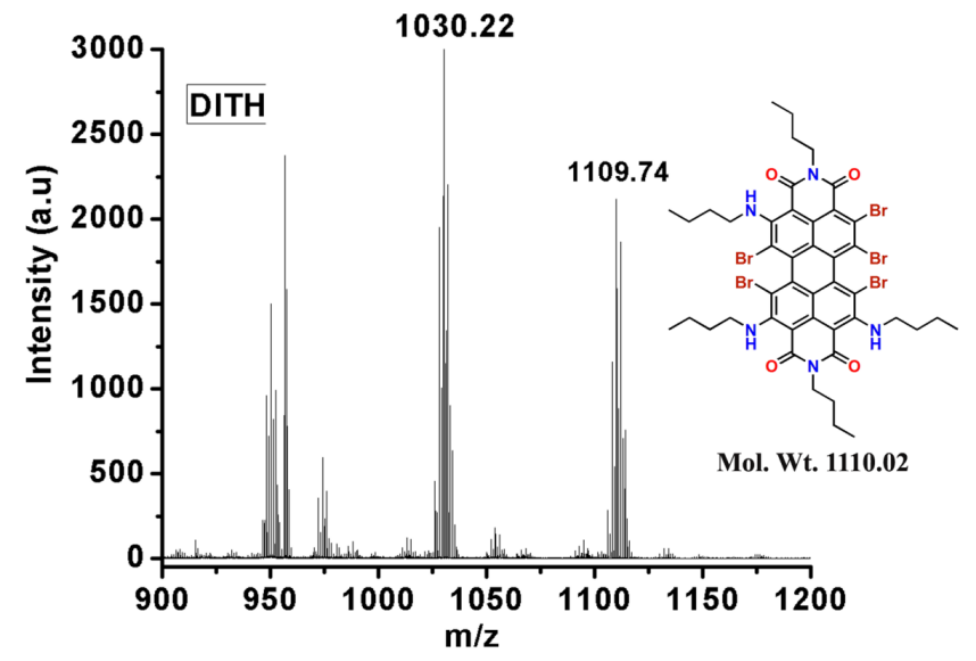

Figure S18: MALDI-TOF mass spectrum of $\mathbf{5 b .}$

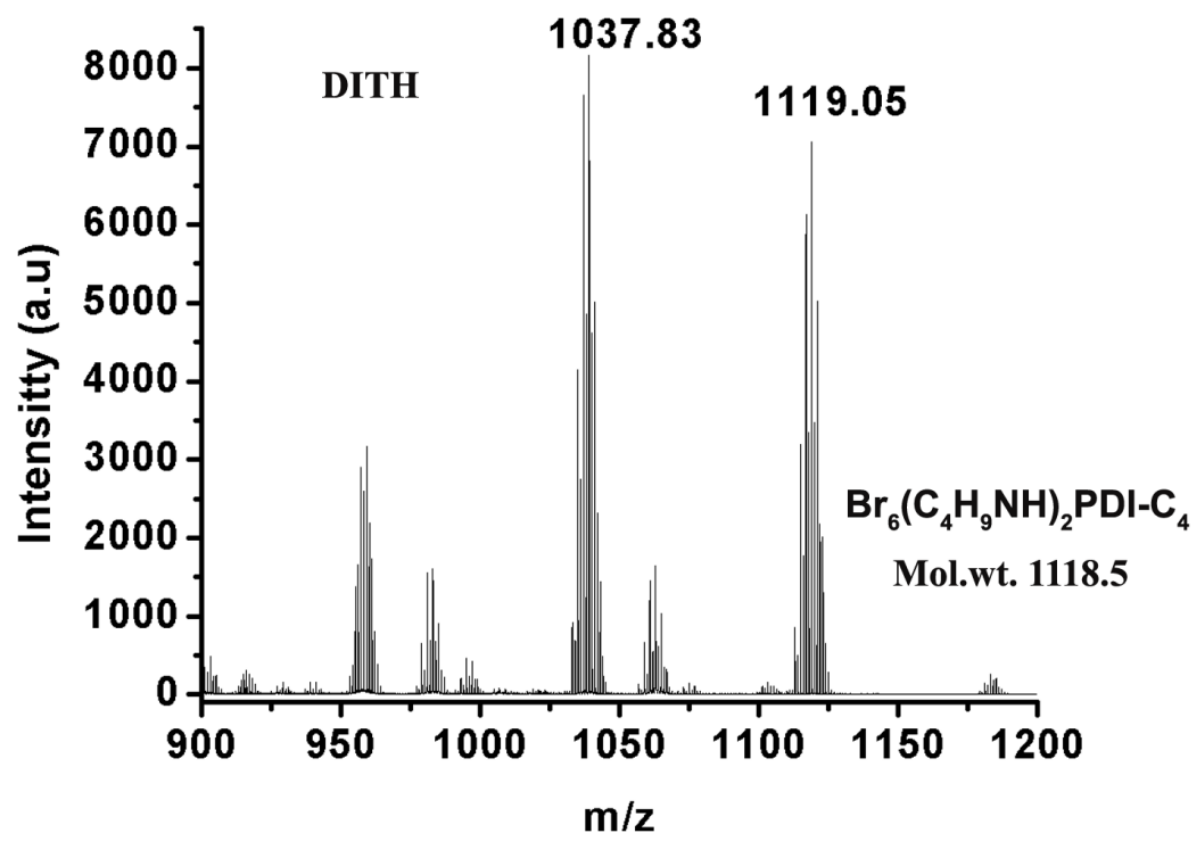

Figure S19: MALDI-TOF mass spectrum of 5c (disubstituted). 


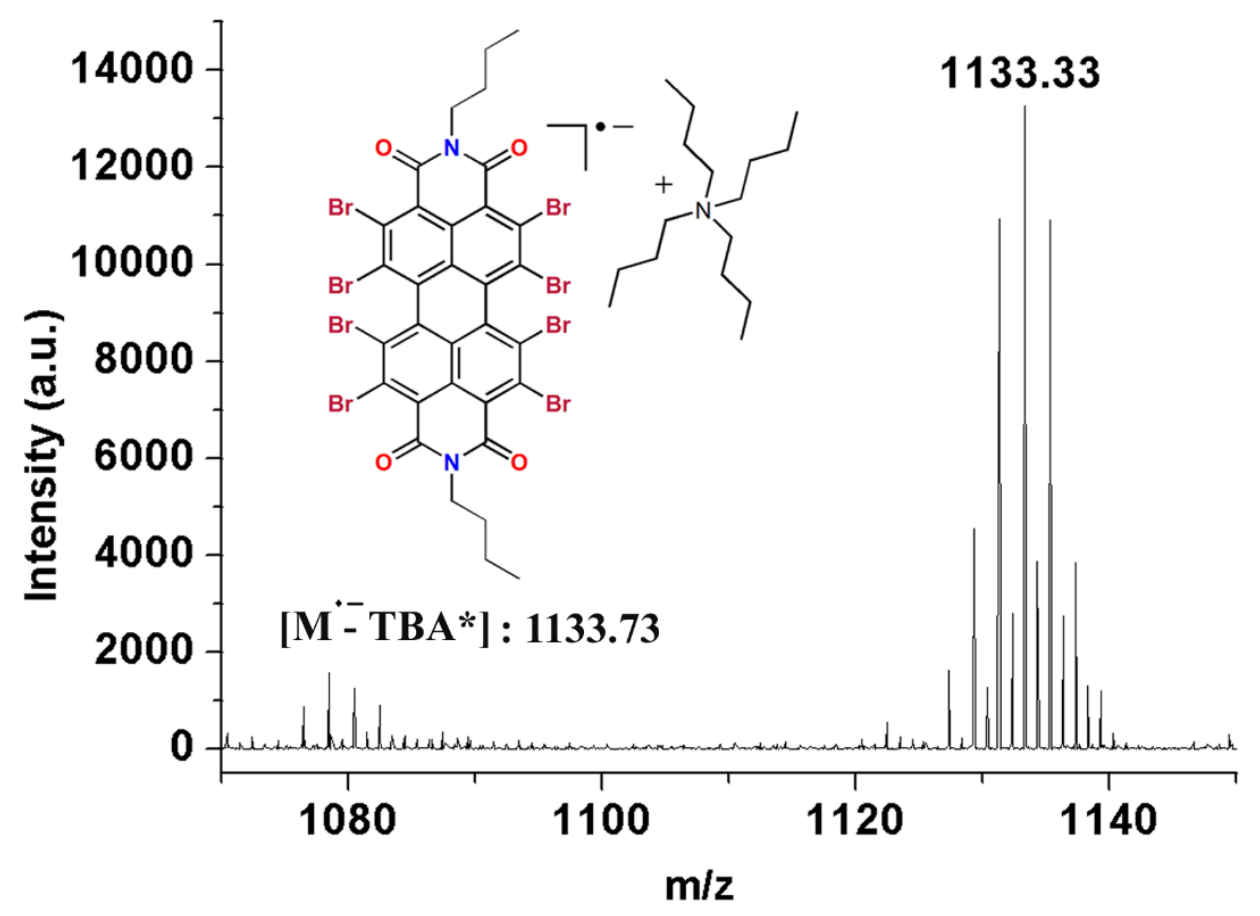

Figure S20: ESI mass spectrum of 2a radical anion. * Tetra butylammonium

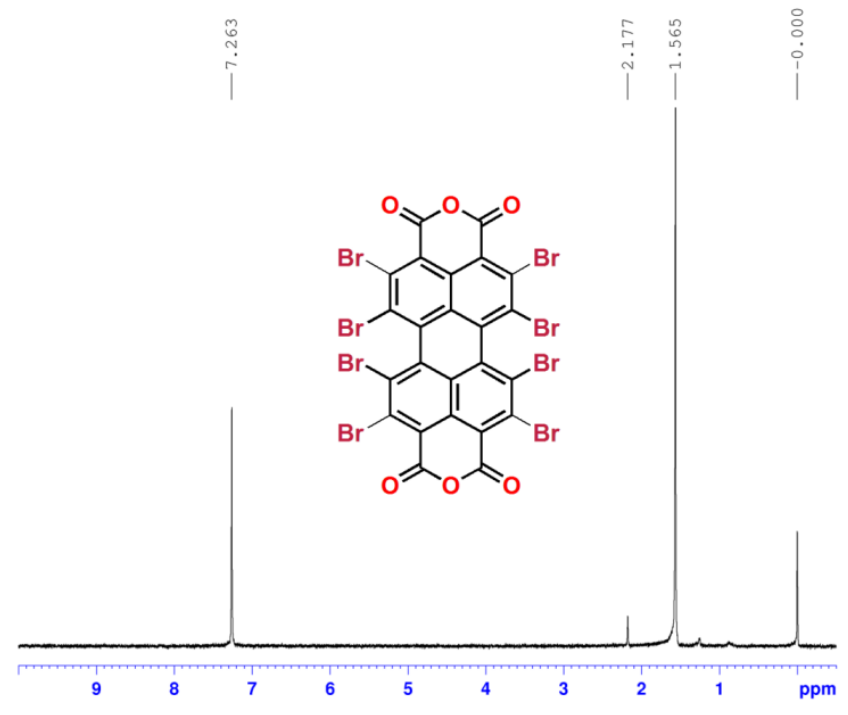

Figure S21: $500 \mathrm{MHz}{ }^{1} \mathrm{H}$ NMR spectrum of 1 in $\mathrm{CDCl}_{3}$ at room temperature. 


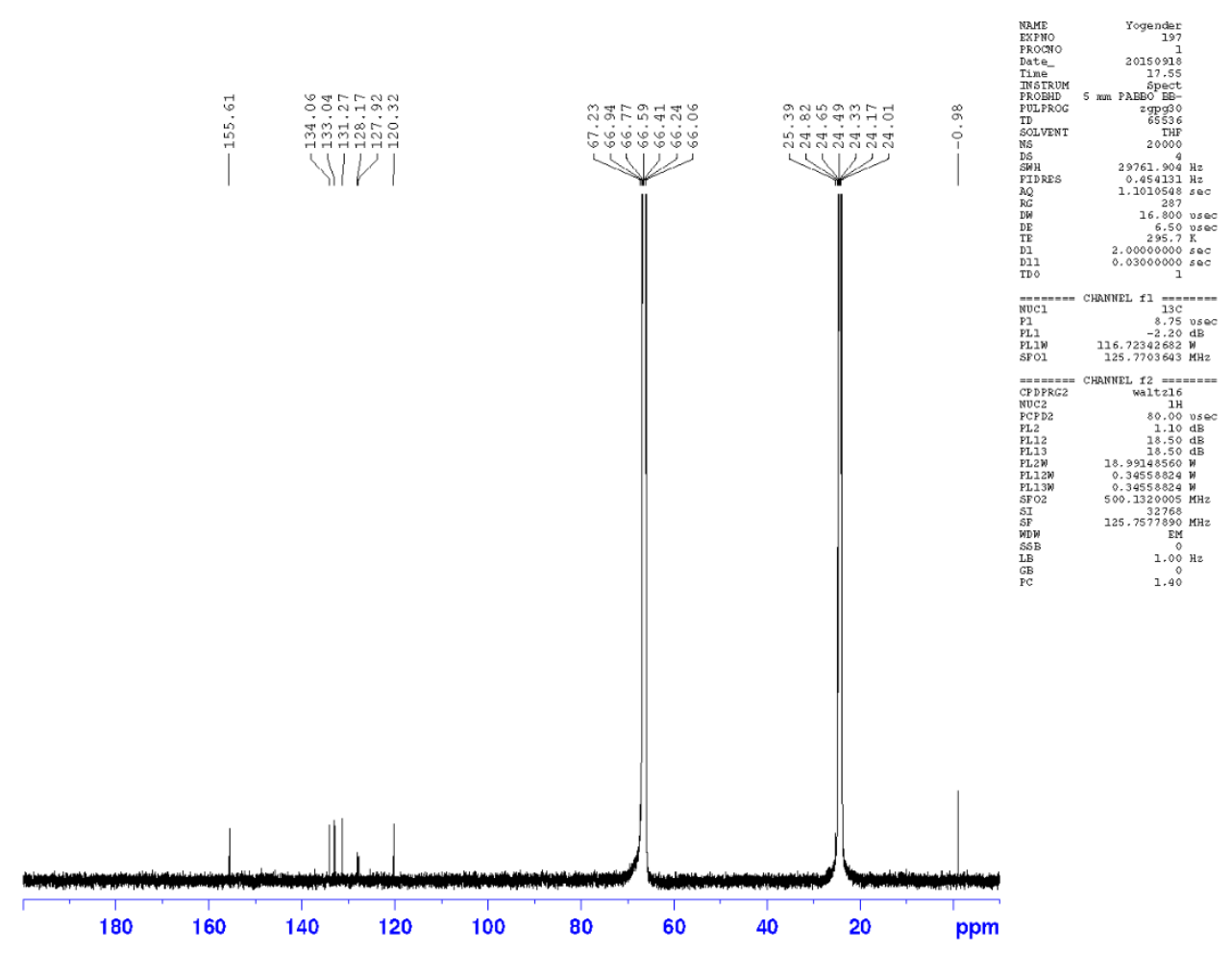

Figure S22: $125 \mathrm{MHz}{ }^{13} \mathrm{C}$ spectrum of $\mathbf{1}$ in $\mathrm{THF}-\mathrm{d}_{8}$ at room temperature.

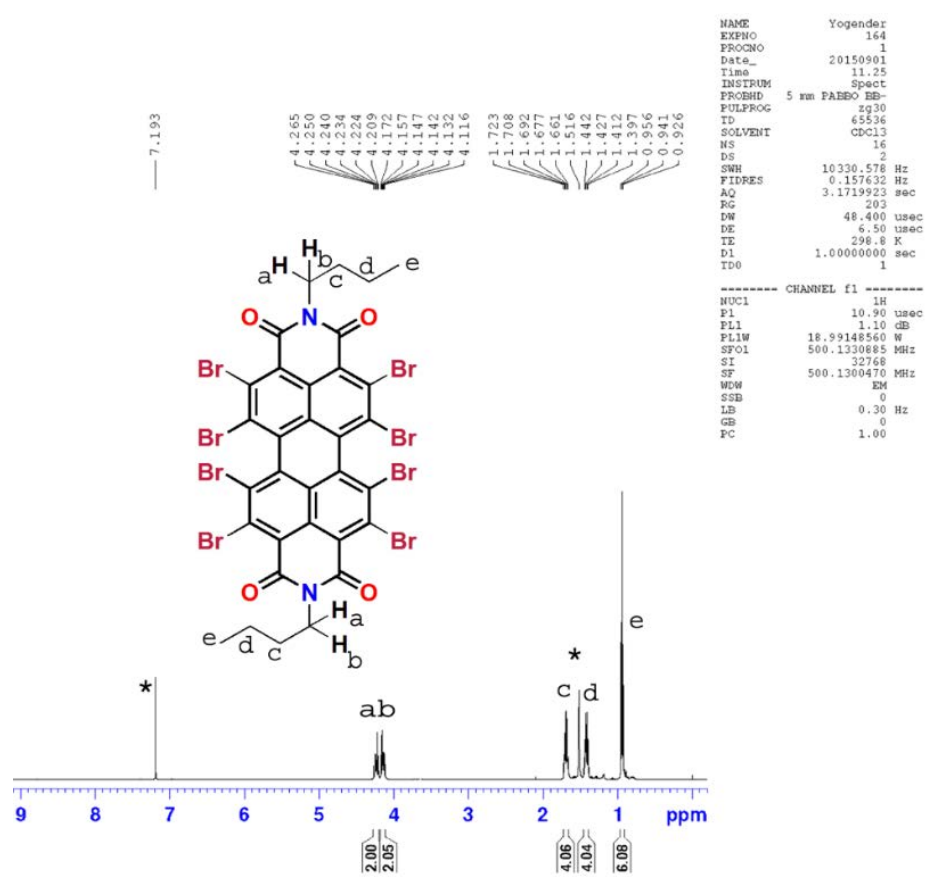

Figure S23: $500 \mathrm{MHz}{ }^{1} \mathrm{H}$ NMR spectrum of $\mathbf{2 a}$ in $\mathrm{CDCl}_{3}$ at room temperature. 


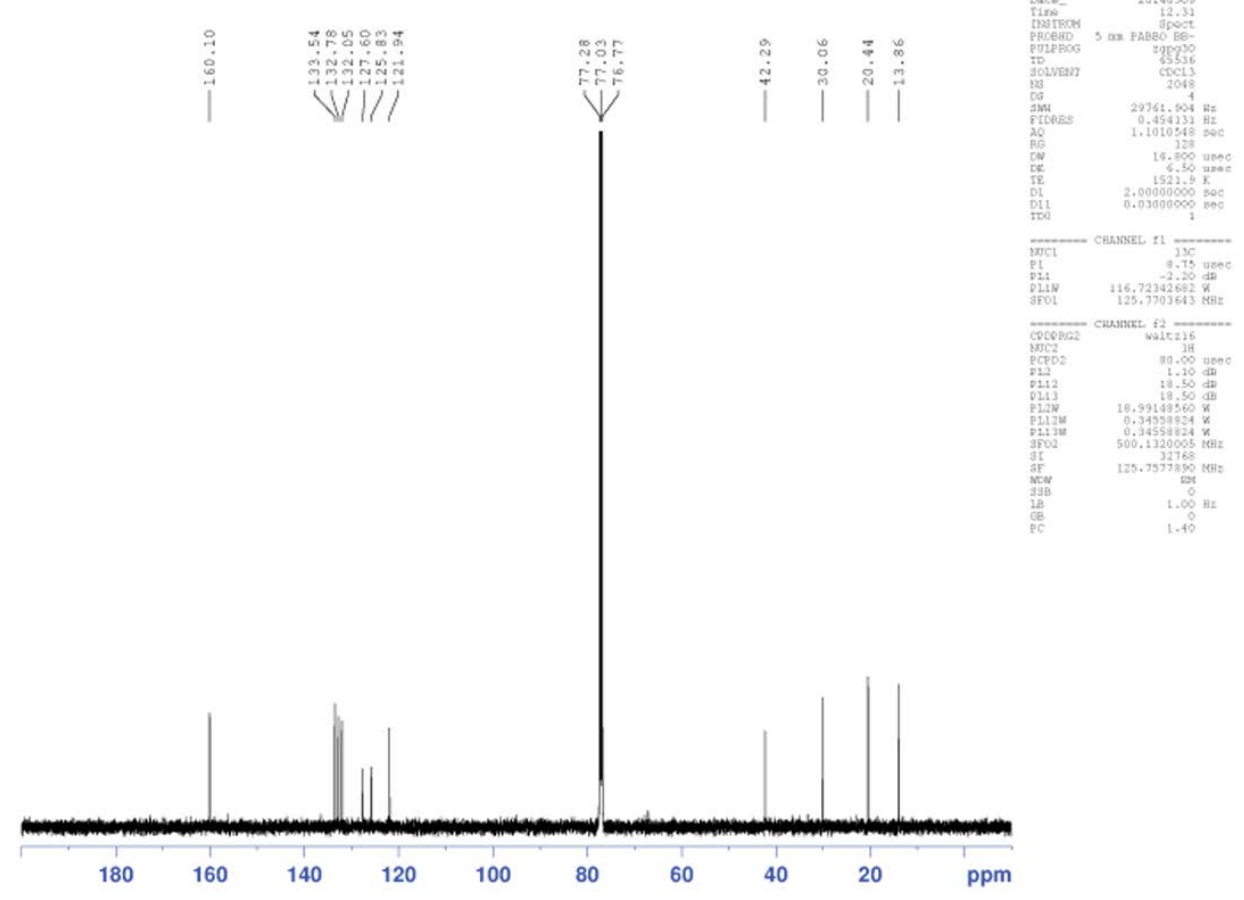

Figure S24: $125 \mathrm{MHz}{ }^{13} \mathrm{C}$ spectra of $\mathbf{2 a}$ in $\mathrm{CDCl}_{3}$ at room temperature.

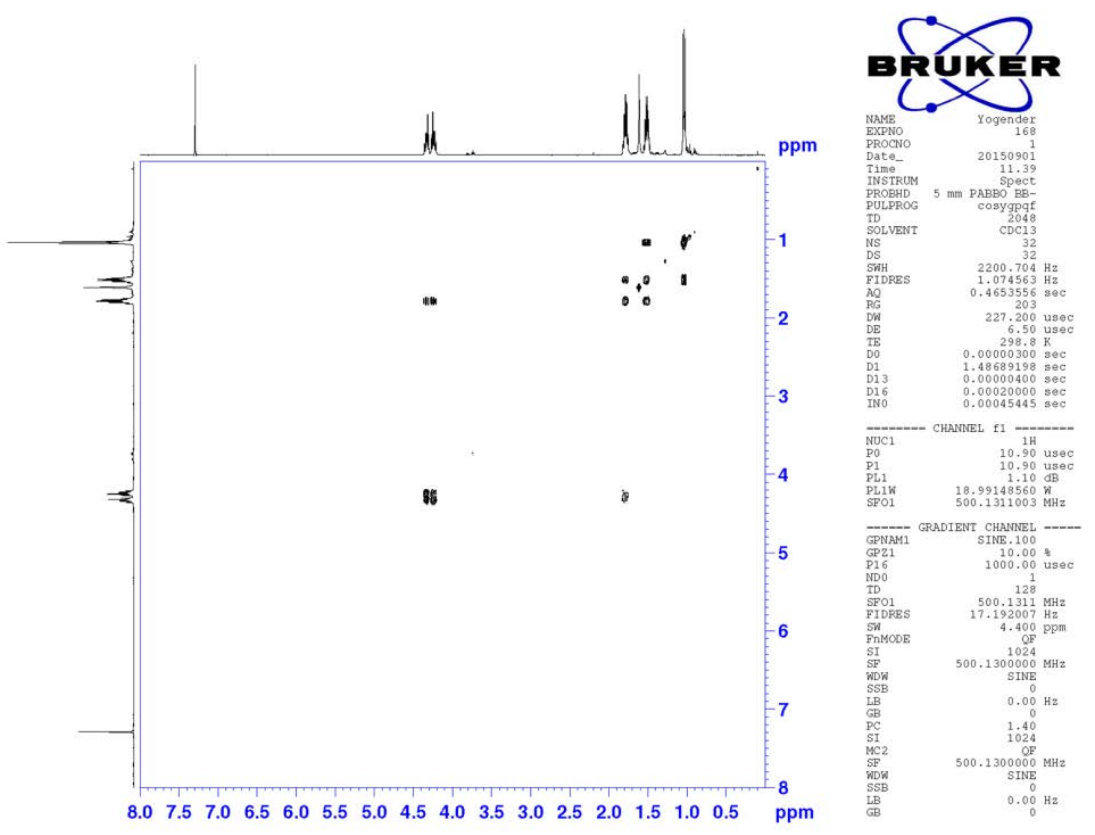

Figure S25: $500 \mathrm{MHz}$ COSY NMR spectra of 2a in $\mathrm{CDCl}_{3}$ at room temperature. 


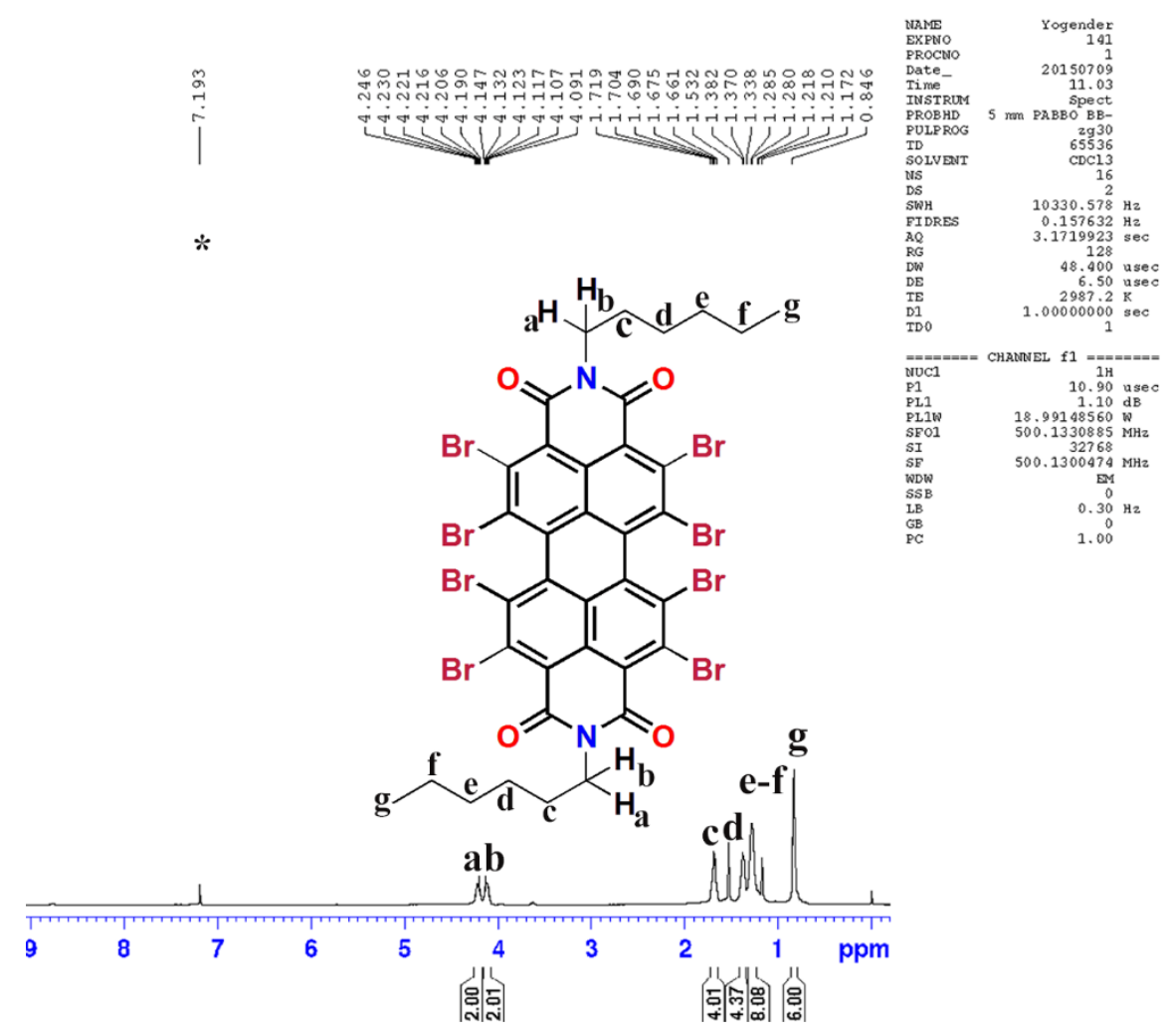

Figure S26: $500 \mathrm{MHz}{ }^{1} \mathrm{H}$ NMR spectrum of $\mathbf{2 b}$ in $\mathrm{CDCl}_{3}$ at room temperature.

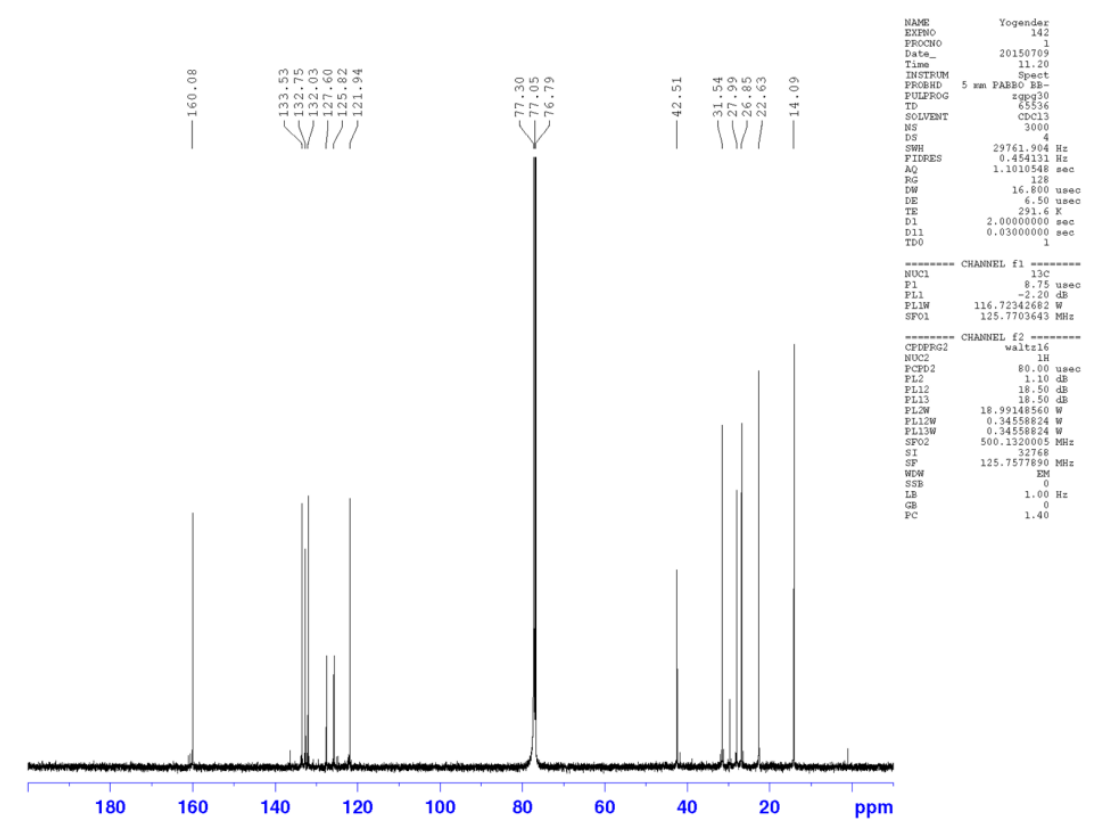

Figure S27: $125 \mathrm{MHz}{ }^{13} \mathrm{C}$ spectra of $\mathbf{2 b}$ in $\mathrm{CDCl}_{3}$ at room temperature. 


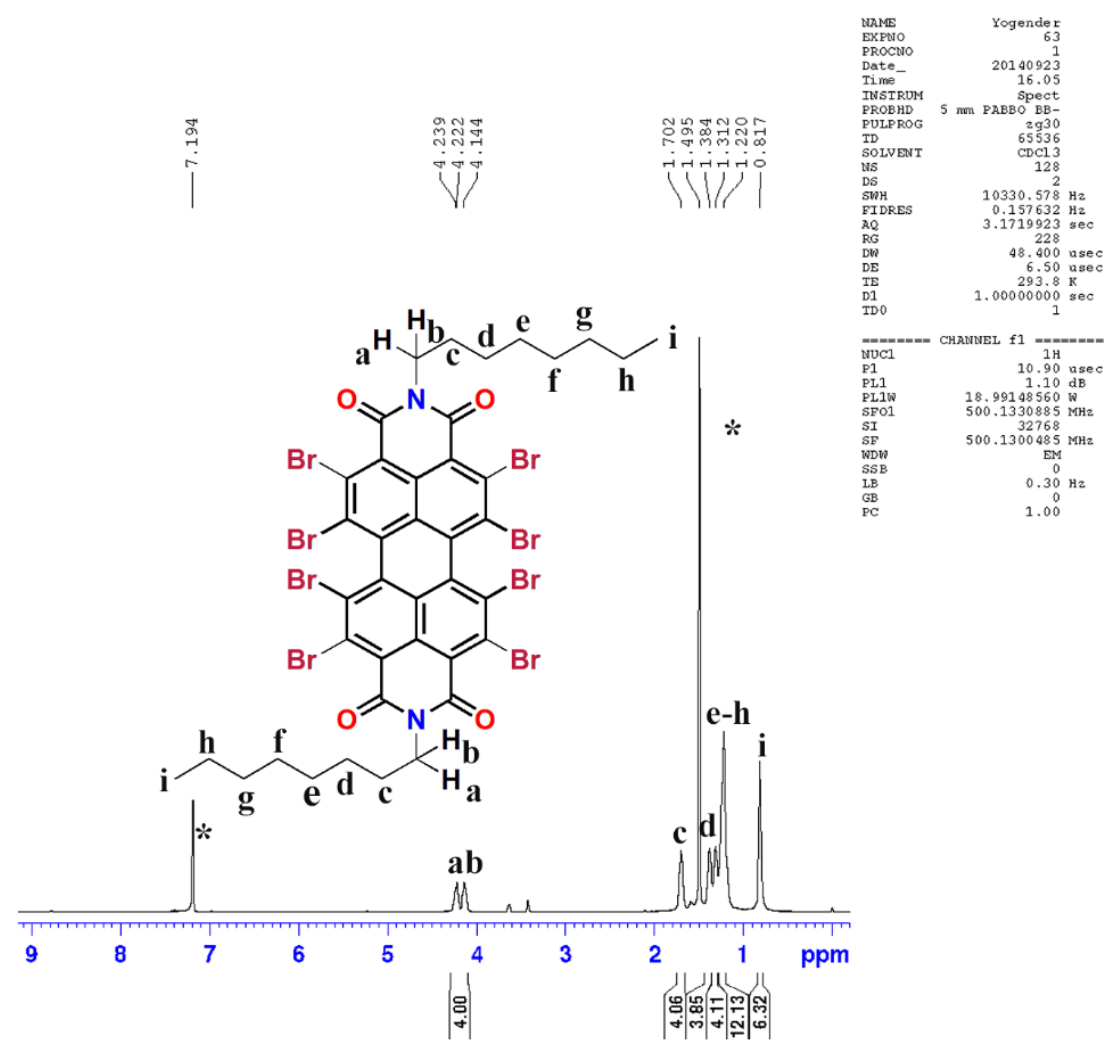

Figure S28: $500 \mathrm{MHz}{ }^{1} \mathrm{H} \mathrm{NMR}$ spectrum of $2 \mathrm{c}$ in $\mathrm{CDCl}_{3}$ at room temperature.

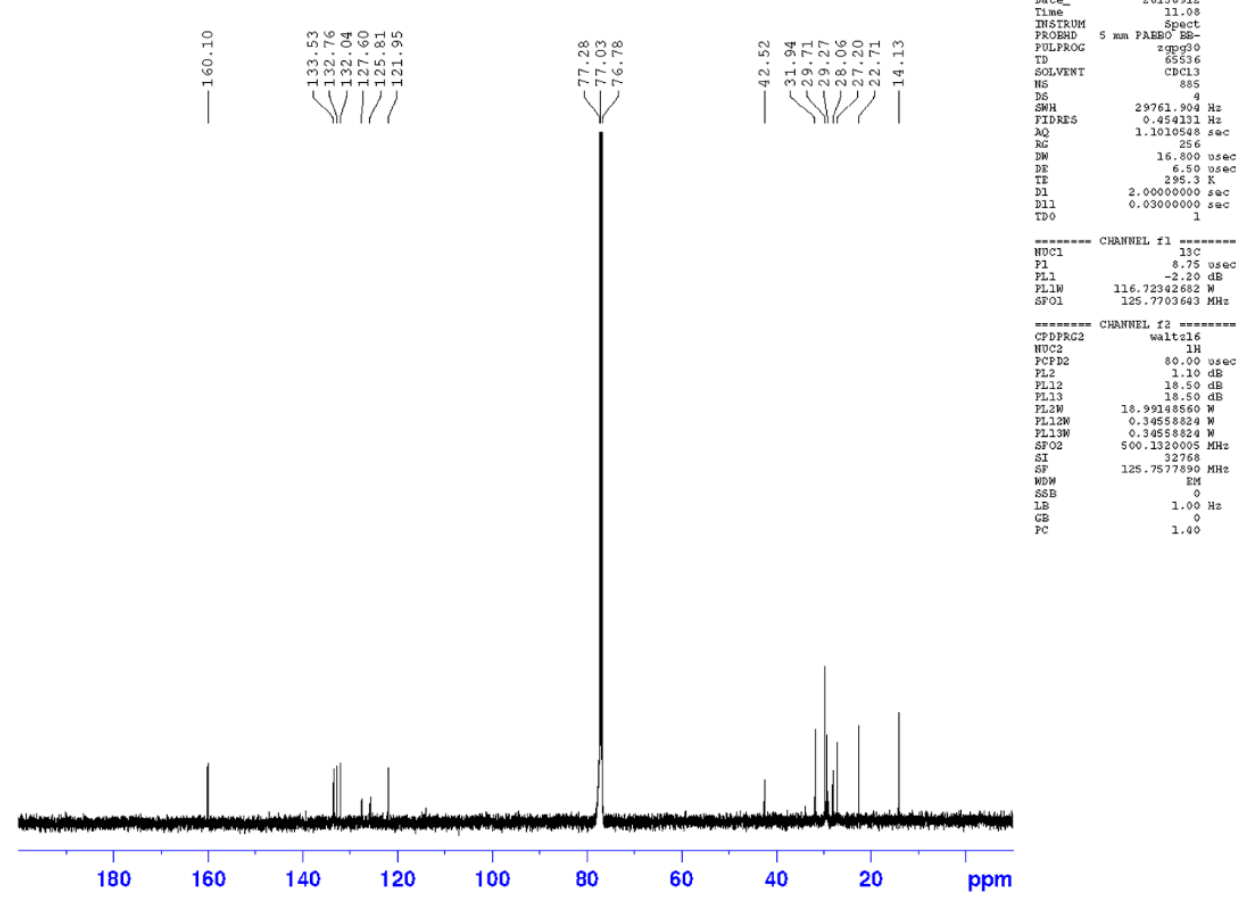

Figure S29: $125 \mathrm{MHz}{ }^{13} \mathrm{C}$ spectra of $\mathbf{2 c}$ in $\mathrm{CDCl}_{3}$ at room temperature. 


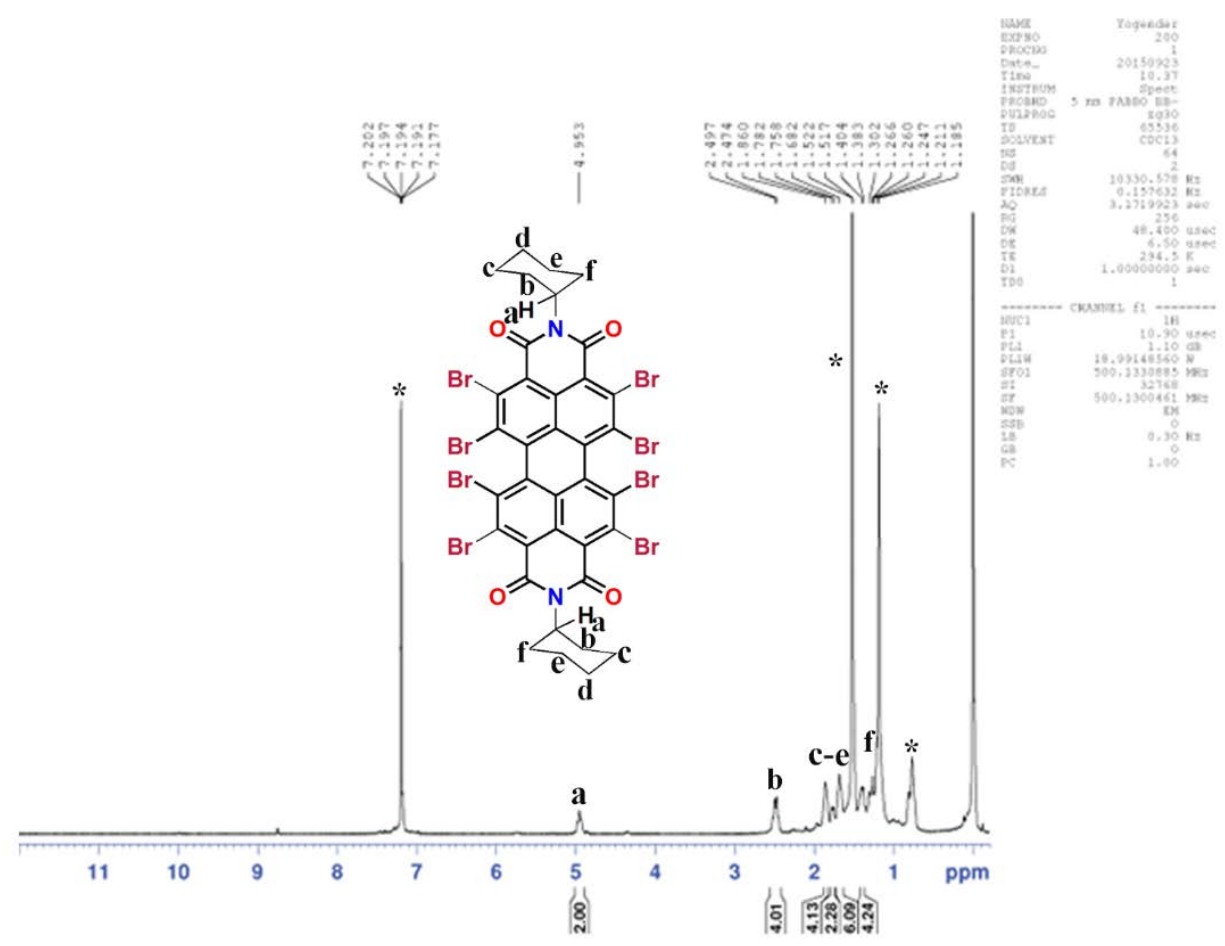

Figure S30: $500 \mathrm{MHz}{ }^{1} \mathrm{H}$ NMR spectrum of $\mathbf{2 d}$ in $\mathrm{CDCl}_{3}$ at room temperature.

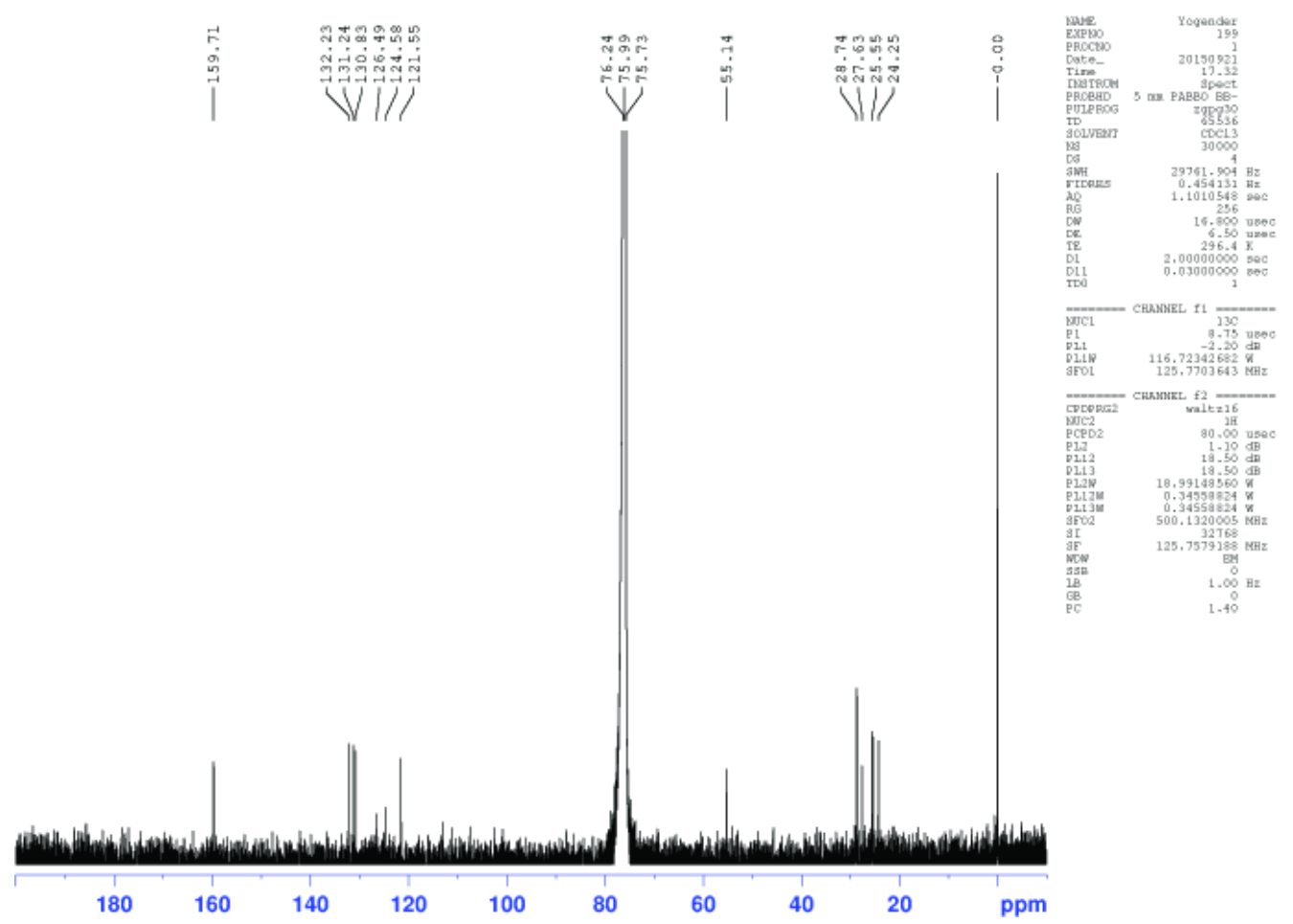

Figure S31: $125 \mathrm{MHz}^{13} \mathrm{C}$ spectra of $\mathbf{2 d}$ in $\mathrm{CDCl}_{3}$ at room temperature. 

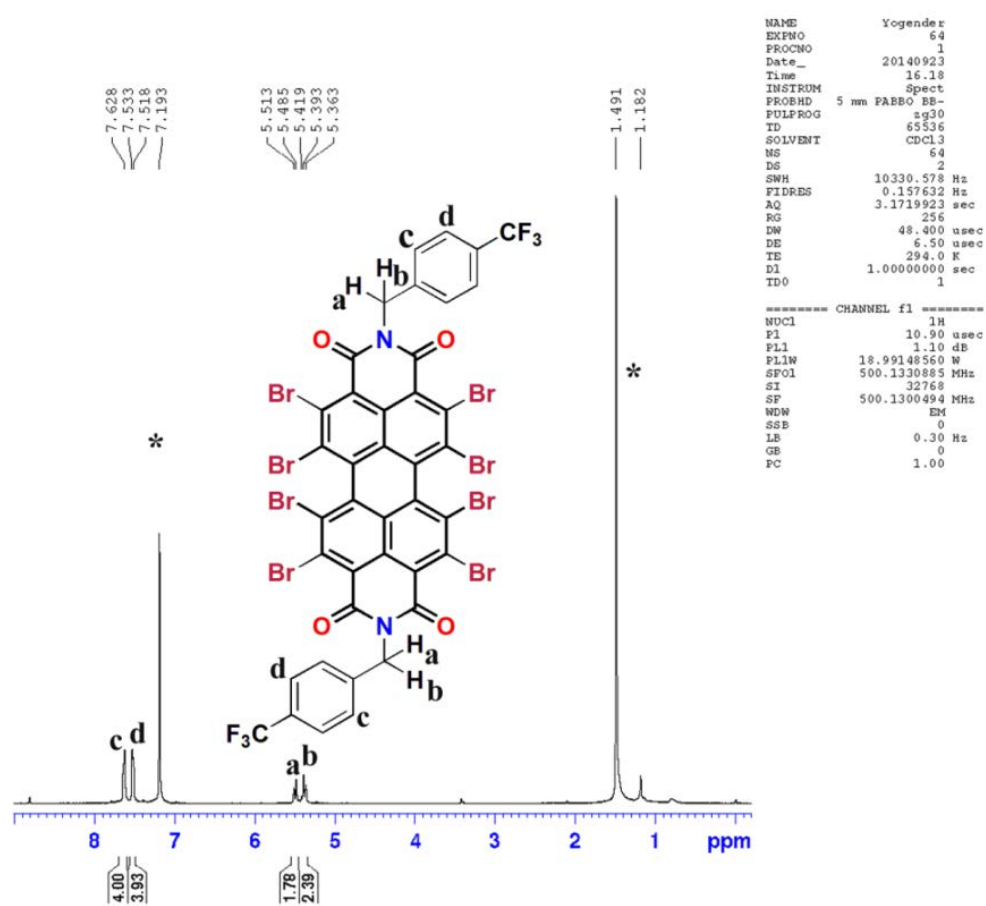

Figure S32: $500 \mathrm{MHz}{ }^{1} \mathrm{H}$ NMR spectrum of $2 \mathbf{e}$ in $\mathrm{CDCl}_{3}$ at room temperature.

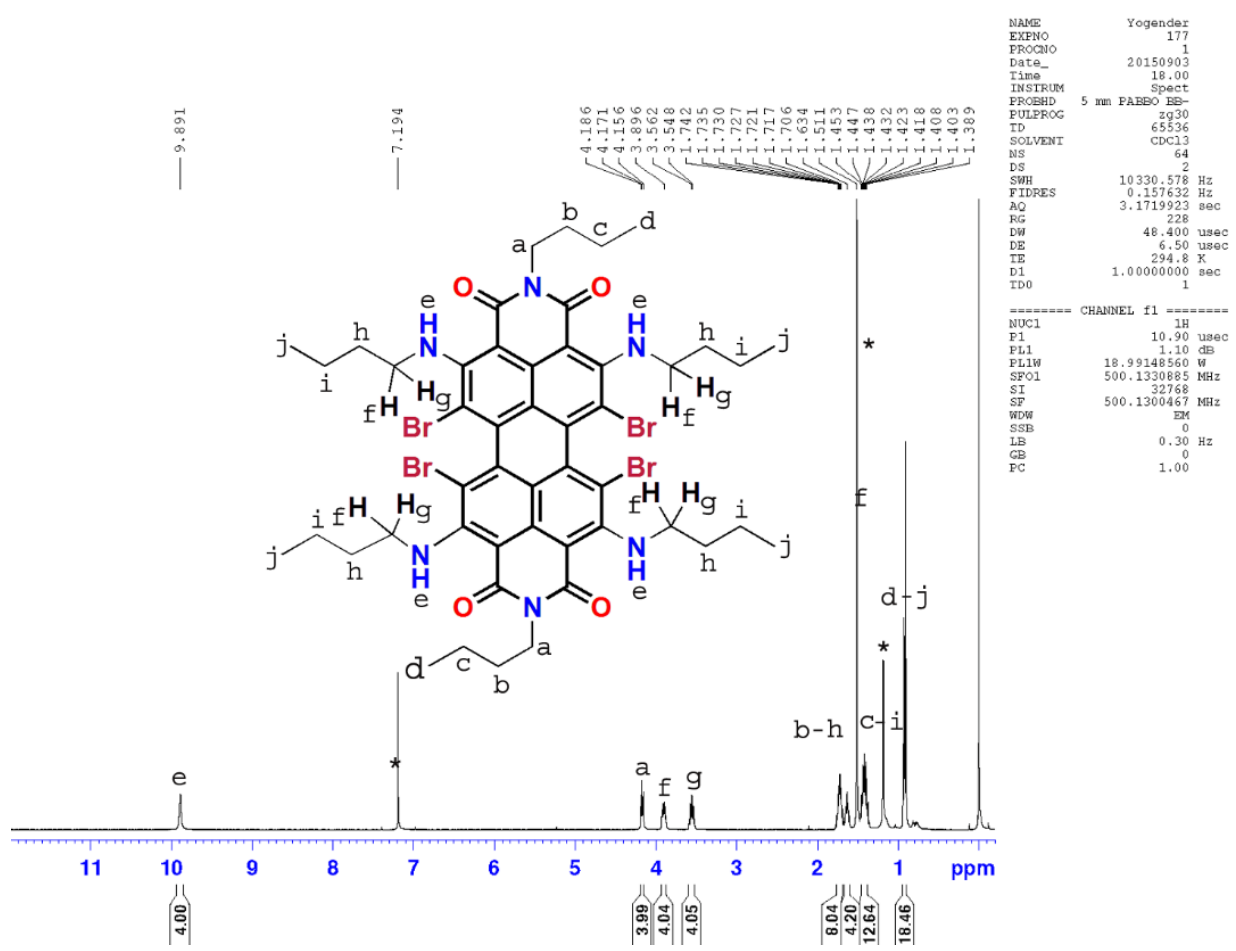

Figure S33: $500 \mathrm{MHz}{ }^{1} \mathrm{H} \mathrm{NMR}$ spectrum of 5 a in $\mathrm{CDCl}_{3}$ at room temperature. 


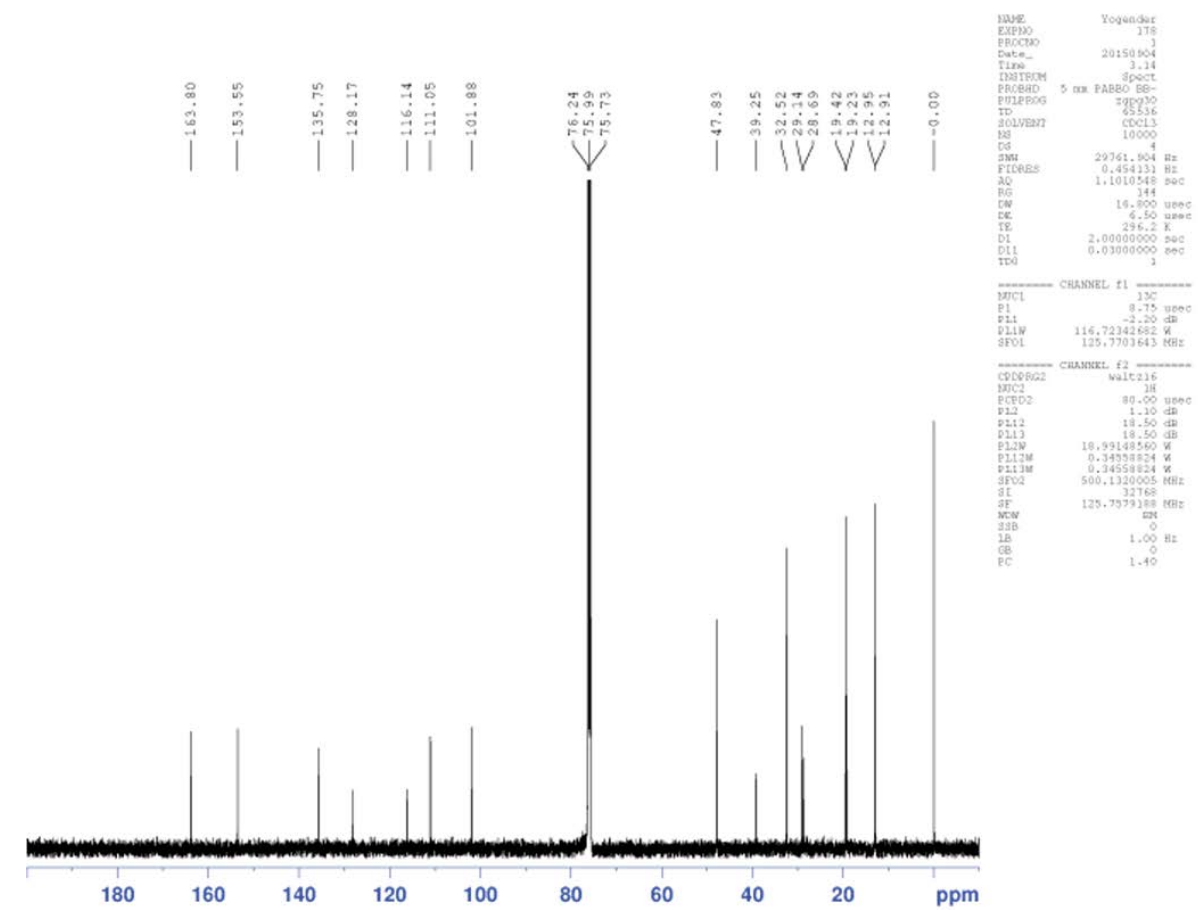

Figure S34: $125 \mathrm{MHz}{ }^{13} \mathrm{C}$ spectra of $5 \mathbf{a}$ in $\mathrm{CDCl}_{3}$ at room temperature.

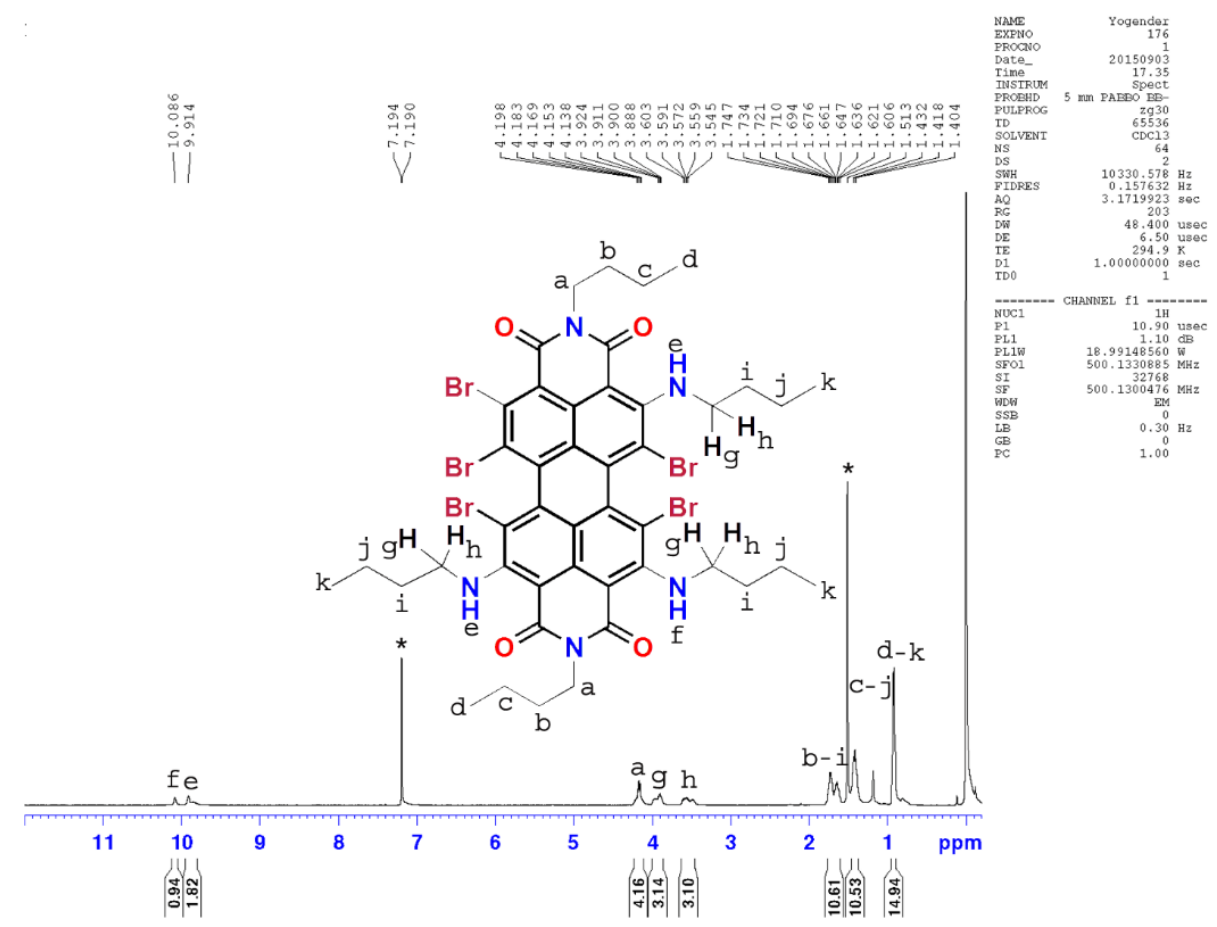

Figure S35: $500 \mathrm{MHz}{ }^{1} \mathrm{H}$ NMR spectrum of $\mathbf{5 b}$ in $\mathrm{CDCl}_{3}$ at room temperature. 


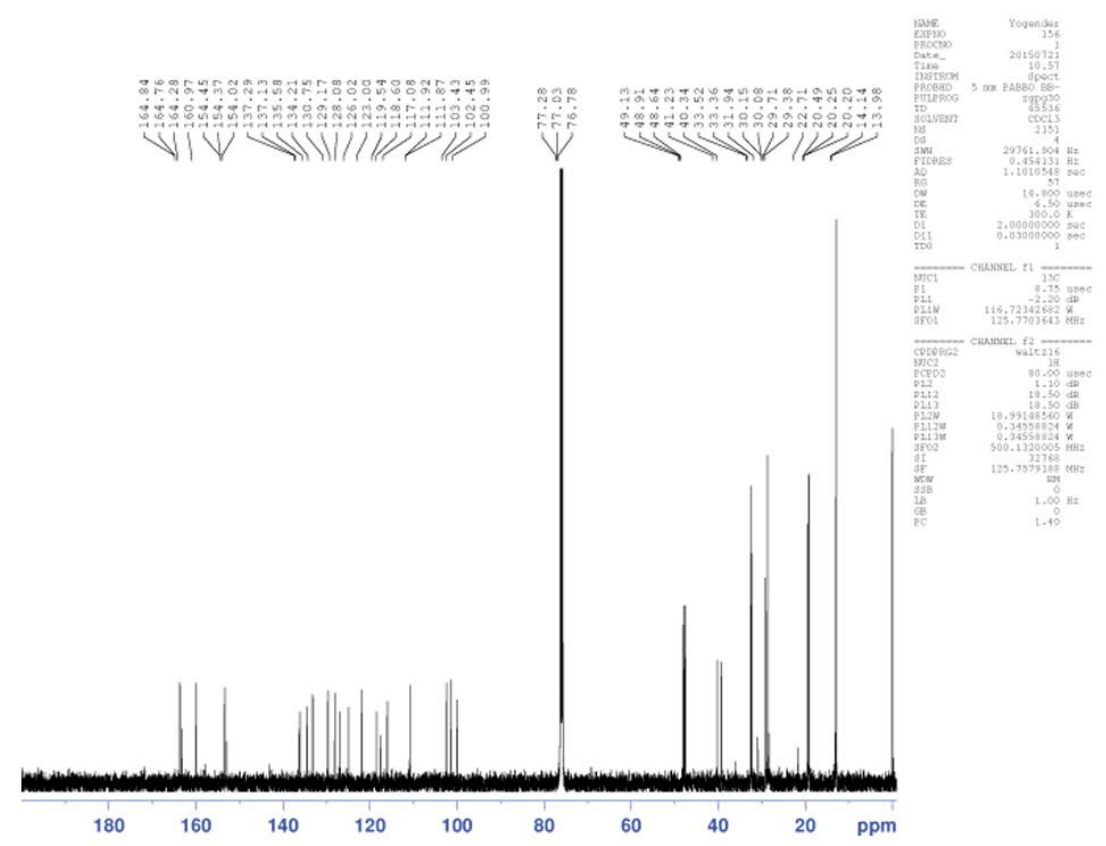

Figure S36: $125 \mathrm{MHz}{ }^{13} \mathrm{C}$ spectra of $\mathbf{5 b}$ in $\mathrm{CDCl}_{3}$ at room temperature.

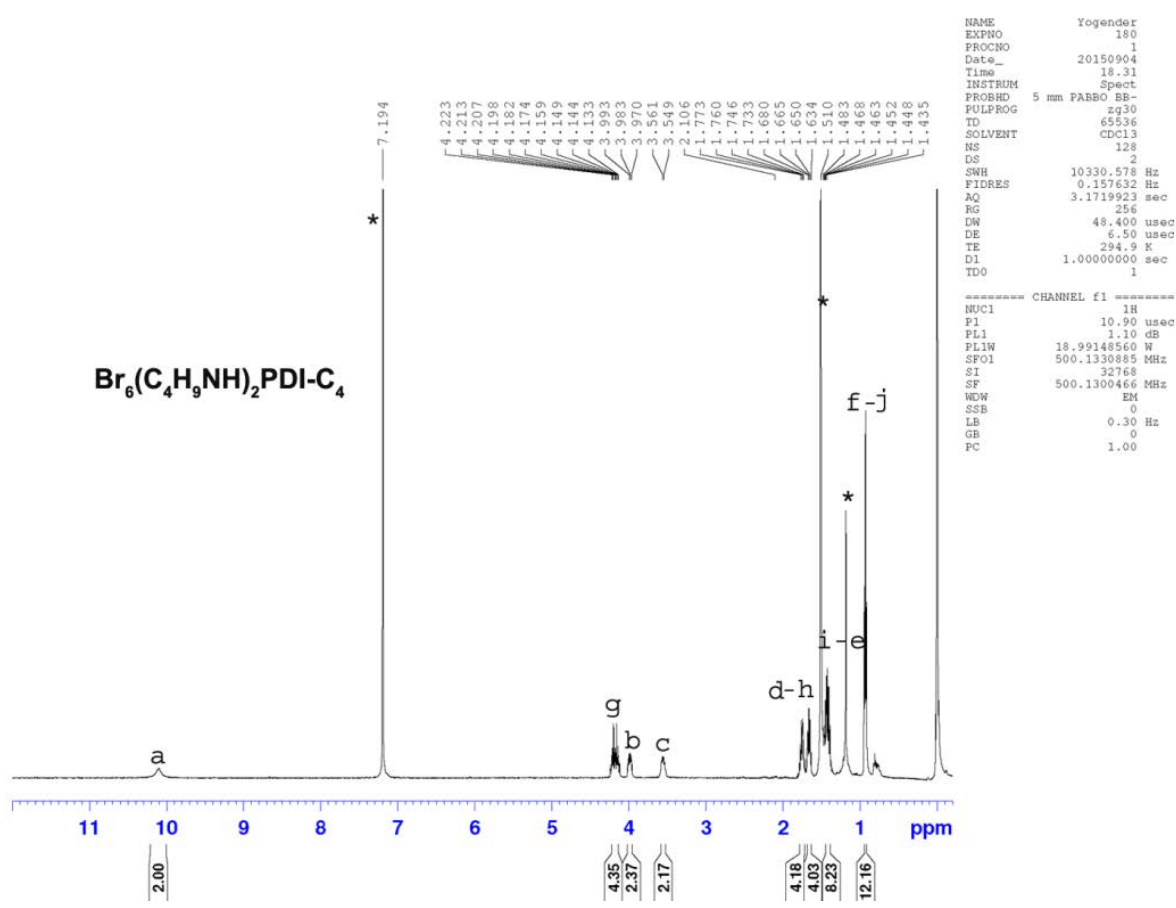

Figure S37: $500 \mathrm{MHz}{ }^{1} \mathrm{H}$ NMR spectrum of 5c (disubstituted) in $\mathrm{CDCl}_{3}$ at room temperature. 


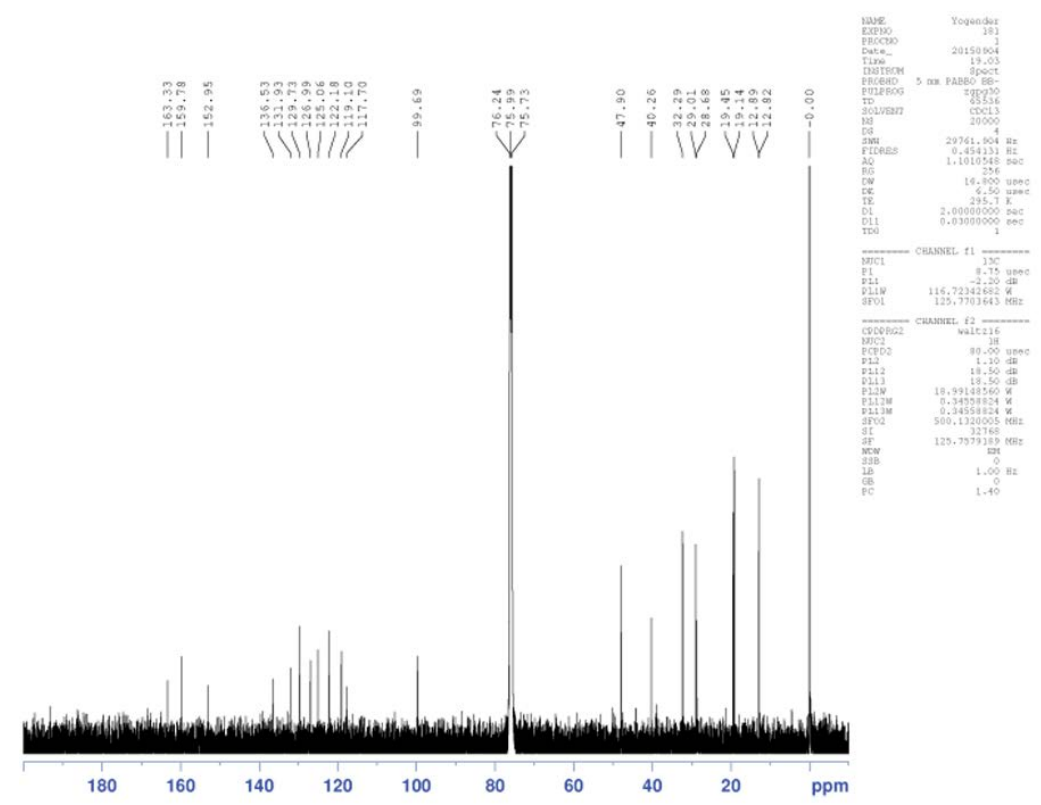

Figure S38: $125 \mathrm{MHz}{ }^{13} \mathrm{C}$ spectra of $5 \mathrm{c}$ in $\mathrm{CDCl}_{3}$ at room temperature.

\section{References:}

1. D. Becke, J. Chem. Phys., 1993, 98, 5648.

2. C. Lee, W. yang, R. G. Parr, Phys. Rev. B, 1988, 37, 785.

3. Gaussian 09, Revision D.01, M. J. Frisch, G. W. Trucks, H. B. Schlegel, G. E. Scuseria, M. A. Rob, J. R., Cheeseman, G. Scalmani, V. Barone, B., Mennucci, G. A. Petersson, H. Nakatsuji, M. Li, X. Caricato, H. P. Hratchian, A. F. Izmaylov, J. Bloino, G. Zheng, J. L. Sonnenberg, M. Hada, M. Ehara, K. Toyota, R. Fukuda, J. Hasegawa, M. Ishida, T. Nakajima, Y. Honda, O. Kitao, H. Nakai, T. Vreven, Jr. J. A. Montgomery, J. E. Peralta, F. Ogliaro, M. Bearpark, J. J. Heyd, E. Brothers, K. N. Kudin, V. N. Staroverov, R. Kobayashi, J. Normand, K. Raghavachari, A. Rendell, J. C. Burant, S. S. Iyengar, J. Tomasi, M. Cossi, N. Rega, J. M. Millam, M. klene, J. E. Knox, J. B. Cross, V. Bakken, C. Adamo, J. Jaramillo, R. Gomperts, R. E. Startmann, O. Yazyev, A. J. Austin, R. Cammi, C. Pomelli, J. W. Ochterski, R. L. Martin, K. Morokuma, V. G. Zakrzewski, G. A. Voth, P. Salvador, J. J. Dannenberg, S. Dapprich, A. D. Daniels, Ö. Farkas, J. B. Foresman, J. V. Ortiz, J. Cioslowski, D. J. Fox, Gaussian, Inc., Wallingford CT, 2009.

4. CrystalClear 2.1, Rigaku Corporation, Tokyo, Japan

5. G. M. Sheldrick, Acta Crystallogr., Sect. A, 2008, 64, 112; SHELX2013.

6. L. Farrugia, J. Wingx suite for small-molecule single-crystal crystallography. J. Appl. Crystallogr., 1999, 32, 837. 
7. Q. Wenfeng, C. Shiyan, S. Xiaobo, L. Yunqi, Z. Daoben, Org. Lett., 2006, 8, 867.

8. F. Würthner, V. Stepanenko, Z. Chen, C. R. Saha-Möller, N. Kocher, and D. Stalke J. Org. Chem., 2004, 69, 7933. 